\title{
Selective Fasciculation and Divergent Pathfinding Decisions of Embryonic Chick Motor Axons Projecting to Fast and Slow Muscle Regions
}

\author{
Louise D. Milner, Victor F. Rafuse, and Lynn T. Landmesser \\ Department of Neurosciences, Case Western Reserve University, Cleveland, Ohio 44106-4975
}

\begin{abstract}
Proper motor function requires the precise matching of motoneuron and muscle fiber properties. The lack of distinguishing markers for early motoneurons has made it difficult to determine whether this matching is established by selective innervation during development or later via motoneuron-muscle fiber interactions. To examine whether chick motoneurons selectively innervate regions of their target containing either fast or slow muscle fibers, we backlabeled neurons from each of these regions with lipophilic dyes. We found that motor axons projecting to fast and slow muscle regions sorted into separate but adjacent fascicles proximally in the limb, long before they reached the muscle. More distally, these fascicles made divergent pathfinding decisions to course directly to the appropriate muscle fiber region. In contrast, axons projecting to different areas of an all-fast muscle did not fasciculate separately and
\end{abstract}

became more intermingled as they coursed through the limb. Selective fasciculation of fast- and slow-projecting motoneurons was similar both before and after motoneuron cell death, suggesting that motoneurons specifically recognized and fasciculated with axons growing to muscle regions containing the appropriate muscle fiber type. Taken together, these results strongly support the hypothesis that "fast" and "slow" motoneurons are molecularly distinct before target innervation and that they use these differences to selectively fasciculate, pathfind to, and branch within the correct muscle fiber region from the outset of neuromuscular development.

Key words: selective fasciculation; selective innervation; motoneuron; muscle; specificity; axon pathfinding; guidance; neuromuscular development; fast-slow innervation
During development, chick motoneurons pathfind specifically and precisely to their muscle targets in the hindlimb (Landmesser, 1992) where they are confronted with a subsequent choice, whether to innervate fast or slow primary myotubes. In mature animals, fast and slow muscle fibers, defined by their speed of contraction, are innervated by "fast" or "slow" motoneurons, the electrical properties of which closely match the contractile properties of the muscle fibers they innervate (for review, see Burke, 1981; Vrbova et al., 1995). Appropriate matching of motoneuron with muscle fiber type is critical for proper muscle function (Kernell, 1992). How this matching is established has been controversial because there are two very different ways it could be achieved (for review, see Thompson et al., 1990). One possibility is that both fast and slow muscle fibers and their innervating motoneurons are molecularly distinct and that matching occurs via molecular recognition and selective innervation. Alternatively, the fast or slow nature of either the muscle fibers or the motoneurons might not be autonomously specified but could be imposed by either the nerve or the muscle fiber after a period of nonselective innervation. In mammals, the lack of known molecular differences that distinguish fast and slow motoneurons at the

Received Jan. 5, 1998; revised Feb. 13, 1998; accepted Feb. 18, 1998.

The study was supported by National Institutes of Health Grants NS 19640 and NS 23678 from the National Institute of Neurological Disorders and Stroke. L.D.M. was supported by National Institutes of Health Training Grant T32 HD07104 from the National Institute of Child Health and Human Development. V.F.R. was supported by the Rick Hansen Man in Motion Legacy Foundation. We kindly thank Roman Fatkhiyev for his technical assistance and Dr. Shilpi Banerjee and Marianne Usiak for critical reading of this manuscript.

Correspondence should be addressed to Dr. Lynn Landmesser, Department of Neurosciences, Case Western Reserve University, 10900 Euclid Avenue, Cleveland, OH 44106-4975.

Copyright (C) 1998 Society for Neuroscience $\quad 0270-6474 / 98 / 183297-16 \$ 05.00 / 0$ time of initial innervation has made it difficult to distinguish between these possibilities.

Cross-reinnervation studies in mature animals have shown that the nerve can alter many of the contractile and metabolic properties of the muscle fiber to match its own functional properties, in large part via its imposed pattern of electrical activity (Buller et al., 1960b; Vrbova et al., 1995; Gordon et al., 1997). This observation together with the lack of molecular markers for young fast and slow motoneurons has led many investigators to favor initial nonselective innervation (for review, see Jolesz and Sreter, 1981). In contrast, the fact that neonatal rodent motor units are strongly biased toward one fiber type (for mouse, see Fladby and Jansen, 1988, 1990; for rat, see Thompson et al., 1984, 1987, 1990; and for rabbit, see Gordon and Van Essen, 1985; Soha et al., 1987; Cramer and Van Essen, 1995) favors selective innervation, as do several other experimental studies in both chick and mammal (Feng et al., 1965; Hnik et al., 1967; Hoh, 1975; Soileau et al., 1987; Vogel, 1987; Vogel and Landmesser, 1987; Rafuse et al., 1996).

Several fortuitous properties of the chick have allowed us to distinguish between these possibilities. In many chick muscles, fast and slow primary myotubes, distinguishable at the earliest stages by molecular markers (Crow and Stockdale, 1986; Fredette and Landmesser, 1991a; for review, see Donoghue and Sanes, 1994), occur in spatially discrete regions (e.g., McLennan, 1983; Fredette and Landmesser, 1991a). Furthermore, their phenotypic differentiation is autonomous to the limb and independent of innervation or electrical activation (Butler et al., 1982; Phillips and Bennett, 1984; Fredette and Landmesser, 1991a,b; for the rat, see also Condon et al., 1990b). Using this system, we have recently reported that when embryonic motoneurons are forced to 
innervate foreign muscles, they selectively innervate muscles or muscle regions containing the muscle fiber type characteristic of their normal target (Rafuse et al., 1996).

To determine whether this selectivity also occurs during normal development, we examine here the innervation of three muscles that contain separate fast and slow regions. We find that motor axons projecting to the fast and slow regions of each muscle fasciculate separately very proximally within the nerve trunks and make unique pathfinding choices to reach the appropriate muscle region. These results strongly support the hypothesis that fast and slow motoneurons are molecularly distinct before target innervation and that they recognize and selectively innervate appropriate muscle fiber types from the outset.

\section{MATERIALS AND METHODS}

Lipophilic dye injections. White Leghorn chicken eggs were incubated for $7-10 \mathrm{~d}$ in a humidified incubator at $39^{\circ} \mathrm{C}$. Embryos were staged according to the method of Hamburger and Hamilton (1951) and then decapitated and dissected in oxygenated Tyrode's solution to expose the lumbosacral spinal cord and hindlimb muscles of the thigh. For retrograde injections, carbocyanine dyes $\mathrm{DiIC}_{18}(3)$ and DiAsp (Molecular Probes, Eugene, OR) were injected through a glass micropipette into the fast or slow muscle regions of either the sartorius (SART), anterior iliotibialis (AITIB), or iliofibularis (IFIB) muscles or into the proximal and distal portions of the all-fast posterior iliotibialis (PITIB) muscle (see Fig. $2 \mathrm{~A}$, schematic, $2 B, C$, whole mounts). India ink was similarly injected into identified dorsal root ganglia to facilitate future identification of the spinal cord segments containing retrogradely labeled motoneuron cell bodies. Embryos were then fixed in $3.7 \%$ formaldehyde-PBS overnight at room temperature, rinsed in PBS, and then incubated in $1 \%$ formaldehyde-PBS in a humidified chamber at $\sim 27^{\circ} \mathrm{C}$ for at least 1 month to allow dye to retrogradely label motoneuron cell bodies in the spinal cord. A total of 69 retrograde dye-injected limbs were used for analysis: 59 at stages 34-36 and 10 at stages 29-31. For orthograde injections, carbocyanine dyes DiI and DiAsp were injected through a glass micropipette into exposed spinal nerve ventral roots (see Fig. $2 A$, schematic). Embryos were fixed and incubated as described above for at least 1 month to allow dye to reach the thigh muscles. A total of 53 limbs were analyzed after orthograde injections: 37 at stages $34-36$ and 16 at stages $29-31$. Muscle whole mounts were then removed from the limb, mounted in PBS between two coverslips, and photographed.

Characterization of intramuscular nerve branching patterns. Nerve branching patterns were visualized in muscle whole mounts after orthograde dye injections (see above) or immunostaining for neurofilament. Muscles chosen for immunostaining were exposed, fixed for $2 \mathrm{~min}$ in cold acetone, rinsed three times in PBS for $5 \mathrm{~min}$ each, and incubated in primary antibody solution (full-strength supernatant of monoclonal antibody $\mathrm{C} 2$ against neurofilament in $0.3 \%$ Triton) for $1.5 \mathrm{hr}$ at room temperature (for more detail, see Dahm and Landmesser, 1988). Muscles were then washed several times in PBS, fixed for $20 \mathrm{~min}$ in $3.7 \%$ formaldehyde-PBS, rinsed again in PBS, and incubated overnight at $4^{\circ} \mathrm{C}$ in fluorescein-conjugated secondary antibody solution [1:100 dilution of goat anti-mouse fluorescein-conjugated secondary antibody (Sigma, St. Louis, MO) in 2\% BSA-PBS]. Finally, muscles were rinsed, mounted between two coverslips in $50 \%$ glycerol-PBS containing $0.03 \mathrm{mg} / \mathrm{ml}$ $p$-phenylenediamine (Sigma) to prevent fading, and photographed.

Frozen sectioning. Dye-injected limbs selected for cryostat sectioning were incubated in $25 \%$ sucrose-PBS overnight at $4^{\circ} \mathrm{C}$ for cryoprotection, mounted in tissue-freezing medium (Triangle Biomedical Sciences, Durham, NC), frozen quickly in dry ice-cooled isopentane, and cryostat sectioned at a thickness of $14 \mu \mathrm{m}$.

Image acquisition and analysis. Patterns of dye labeling were first analyzed and photographed (Kodak Tri-X Pan film) in whole embryos on an inverted Nikon Diaphot 300 microscope equipped with epifluorescence. After cryostat sectioning, sections were viewed with epifluorescence on an upright Nikon Microphot-FX microscope equipped with a Javelin Ultrichip CCD camera (Javelin Electronics, Los Angeles, CA). Images were acquired using different filter cubes and stored directly onto a computer using the Argus Hamamatsu Image Processor (Hamamatsu Photonics K.K.) in series with the Metamorph Imaging System (Universal Imaging Corporation, West Chester, PA). Acquired images were color encoded and superimposed with the Metamorph system and sub- sequently cropped in Adobe Photoshop to generate the figures. Some figures were subsequently printed in black and white by converting the color-encoded image to gray scale before printing. Modifications to the acquired digital images were limited to color encoding and changes in brightness, contrast, and cropping. Digital images of intact embryos were acquired on the inverted Nikon Diaphot 300 microscope using the same CCD camera and image analysis equipment.

Camera lucida drawings were made by tracing the spinal cord and motoneuron cell bodies that were viewed under epifluorescence on the Nikon Microphot-FX microscope and projected onto a monitor through the CCD camera and Argus image processor (see above).

Muscle whole mounts labeled either with dye or with immunostaining were photographed using Kodak Tri-X Pan film on the Nikon MicrophotFX. Photographic montages of the muscle were then constructed.

Quantification of the segmental distribution of fast- and slow-projecting motoneurons. A total of 44 stage 34-36 retrogradely labeled limbs was used to estimate the proportion of fast- and slow-projecting motoneurons from each spinal cord segment of a motor pool. Only those limbs in which dye brightly labeled the spinal nerves were used for analysis. Because lipophilic dyes labeled only those motoneurons projecting to the site of injection, not all neurons in the motor pool were labeled after muscle injection. Three counting methods were used to quantify motoneuron distributions. (1) In seven embryos, spinal cords were sectioned, and brightly labeled cell bodies in the lateral motor column of every third section (each section is $14 \mu \mathrm{m}$ thick) were counted. From these raw counts, the proportion of the total number of DiI-labeled cells that were located in each segment was calculated. The same calculation was determined for the DiAsp-labeled cells. Approximately 100 cells were labeled in each motor pool without using a correction factor. Motor pools in a minimum of two embryos for each muscle were counted in this manner. (2) In most embryos, the proportion of labeled axons in each spinal nerve was also estimated in intact embryos. Because the precise number of axons could not be counted directly, we visually estimated the proportion of the total number of DiI-labeled axons that were in each spinal nerve to the nearest $5 \%$. In cases in which only a few axons were labeled, the segment was scored as $<5 \%$. In cases in which axon fasciculation was extensive, a range was often used to express the proportion of labeled axons. DiAsp-labeled axons were estimated in a similar manner. (3) Finally, in some embryos, distinct motoneuron cell bodies were visible in the intact spinal cord (e.g., see Fig. $2 B, C$, black arrows), and counts of DiI- and DiAsp-labeled cells were made. Calculations of the proportion of labeled cells in each segment were again made separately for DiI- and DiAsp-labeled cells to normalize for differences in DiI versus DiAsp labeling. The data (see Fig. 4, bar graphs) represent the mean proportion of labeled neurons projecting from each segment to the fast or slow regions of the muscle, as determined by one or more of the above methods of counting $( \pm \mathrm{SE})$. In embryos in which multiple counting methods were used, the data for that embryo were averaged separately before being pooled with data from other embryos. The segmental distribution of motoneurons (see Fig. 8) for two stage 30-31 embryos was generated by visually estimating the proportion of labeled axons in each spinal nerve similar to counting method 2 described above for older stage 34-36 embryos.

Cresyl violet staining. After image analysis, some sections from dyelabeled embryos were stained with cresyl violet to confirm the position and size of the nerve trunks in the limbs.

Electromyograms. In vitro spinal cord-hindlimb preparations were performed as described previously (Landmesser and O'Donovan, 1984a; Rafuse et al., 1996) to record electromyograms (EMGs) from the fast and slow regions of stage 36 SART, AITIB, and IFIB muscles. Briefly, embryos were decapitated quickly and placed in cold oxygenated Tyrode's solution. A ventral laminectomy was performed, and the limb was dissected to expose the muscles of interest. The preparation was incubated in well-oxygenated Tyrode's solution at $28-30^{\circ} \mathrm{C}$ for several hours, after which the motoneurons became spontaneously active. Muscle activation patterns were recorded simultaneously from the fast and slow regions of each muscle using small polyethylene suction electrodes pulled from polyethylene tubing (PE-190; Clay Adams, Parsippany, NJ). After stimulation to the thoracic spinal cord [using single-pulse electrical stimuli that were generated from a Grass S88 stimulator (Grass, Quincy, MA) and isolated from ground with a Grass PSIU6B stimulator isolation unit], each muscle gave a series of bursts that were visualized on an oscilloscope (R5030; Tektronix, Beaverton, OR) and brush recorder (Gould, Cleveland, OH) and were recorded on an analog tape (Vetter, Rebersburg, PA) for further analysis.

Electrophysiological data analysis. EMG activation patterns were digi- 

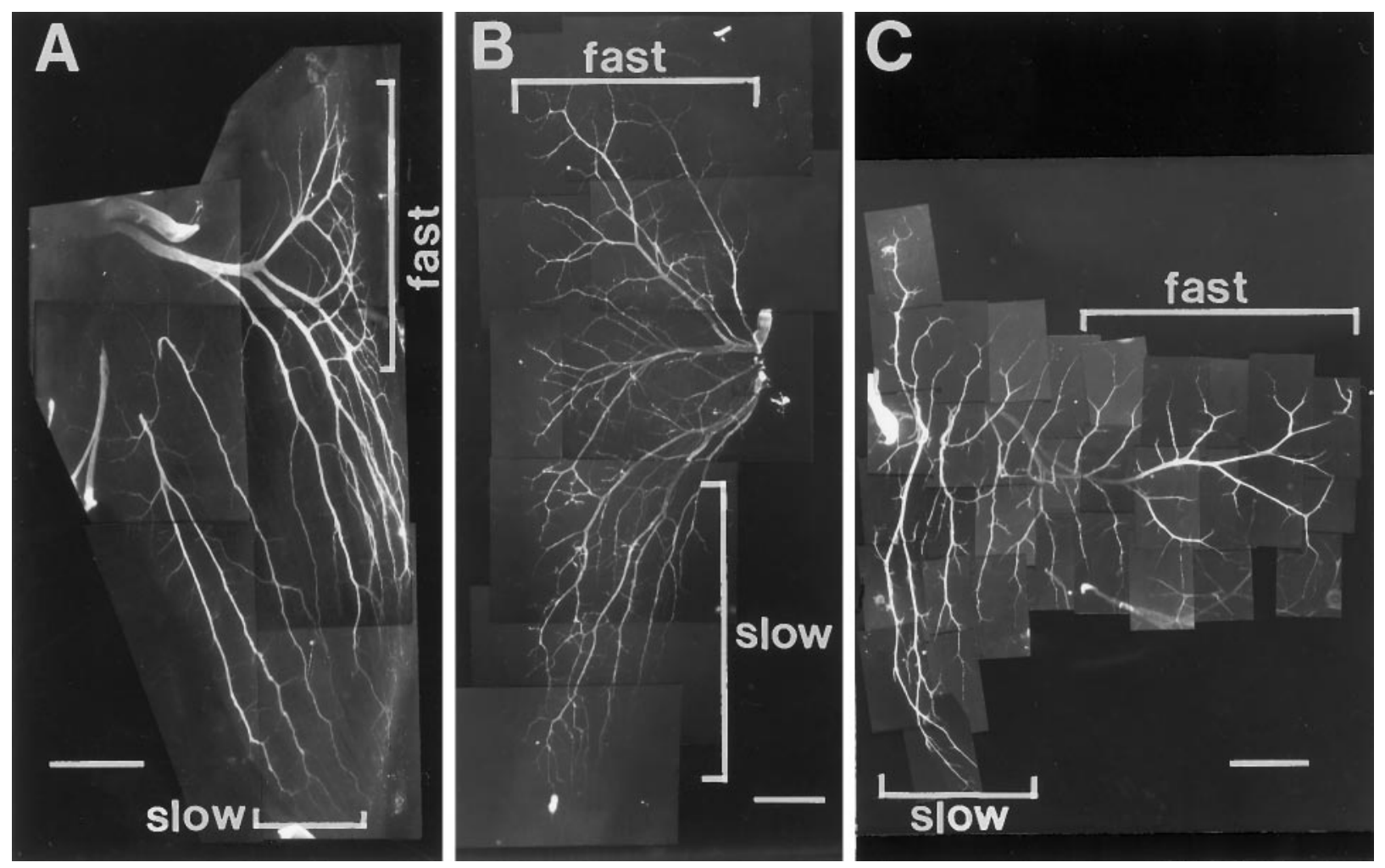

Figure 1. Intramuscular nerve branching patterns of three hindlimb muscles with separate fast and slow muscle regions. Muscle whole mounts of IFIB $(A)$, SART $(B)$, and AITIB $(C)$ at stage 36 were stained with anti-neurofilament antibody to visualize the nerves. $A-C$, Anterior is to the left, proximal is at the top, and myotubes are oriented top to bottom. A, Nerves in the slow region of the IFIB grew parallel to the myotubes (lower bracket) and sent off multiple collateral sprouts at regular intervals. In contrast, axons in the fast region grew transversely across the myotubes before branching in a "reductive" pattern to innervate focally the myotubes (upper bracket). B, Motor axons entered the SART at the posterior muscle edge and grew proximally to reach the fast region (upper bracket) or distally to innervate the slow region (lower bracket). Despite the proximal and distal orientation of the muscle regions, axon branching patterns resembled those in the IFIB. Axons innervating the slow SART grew predominantly along the myotubes (lower bracket), sending off small branches, whereas axons in the fast region first grew across the myotubes and branched reductively (upper bracket). $C$, Axons in the slow AITIB (lower bracket) entered the muscle and grew longitudinally along the muscle fibers, sending off multiple branches similar to those in the IFIB. In the fast region (upper bracket), axons grew perpendicular to the myotubes and then branched sparsely in a reductive pattern. Scale bars: $A, B, 500 \mu \mathrm{m} ; C, 1 \mathrm{~mm}$.

tized with an analog-to-digital converter (GW Instruments, Inc., Somerville, MA) and analyzed with SuperScope II software (GW Instruments, Inc.). EMG activation patterns were quantified as described previously (Landmesser and O'Donovan, 1984b). Briefly, muscle activity was measured in cycles with each cycle beginning at the onset of synchronous EMG discharge (e.g., see Fig. $9 A$, time 0 in histograms). Cycles were divided into $40 \mathrm{msec}$ intervals, and the proportion of time the muscle was active during each $40 \mathrm{msec}$ interval of the cycle was used to construct a frequency histogram. EMG activity preceding the onset of each cycle was only included in the frequency histograms if it was active immediately before the onset of the synchronous discharge. Consequently, the frequency histograms describe the probability of a given muscle being active at any given time preceding, and after, the onset of each cycle.

\section{RESULTS}

\section{Motoneurons grow and branch differently in fast and slow muscle regions}

In addition to the spatial segregation of fast and slow primary myotubes, avian fast and slow muscle fibers exhibit different patterns of innervation; slow fibers are innervated at multiple sites along each myotube, whereas fast fibers are innervated at a single site (Ginsborg and Mackay, 1961). These differences in innervation correlate with striking differences in the pattern of axon ingrowth and branching in fast and slow muscle regions. The intramuscular nerve branching pattern for the three muscles characterized in this study are shown in Figure 1 as neurofilament-stained whole mounts. In all cases, muscle fibers run from top to bottom. In the IFIB muscles (Fig. $1 A$ ), the main intramuscular nerve trunks grow into the slow region (lower bracket) parallel to the muscle fibers. As described previously in more detail (Dahm and Landmesser, 1988), these nerve trunks subsequently send off collateral sprouts at regular intervals to produce the pattern of distributed synapses characteristic of avian slow (tonic) muscle fibers. In contrast, axons in the fast region grow transversely across the myotubes and then branch in a reductive pattern (Fig. 1A, upper bracket) to establish the focal innervation characteristic of fast muscle. Establishing proper branching is crucial for the function of muscle, because chick slow muscle fibers, unlike fast fibers, do not normally conduct action potentials and thus need to be activated at multiple sites to contract effectively (Hnik et al., 1967).

Similar whole mounts were used to characterize the other two muscles used in this study. In both the SART (Fig. $1 B$ ) and the AITIB (Fig. 1C) muscles, nerve trunks in the slow region grew predominantly parallel to the myotubes along most of their length (lower brackets) and sent off collateral sprouts. In contrast, growth in the fast region was oriented predominantly transverse to the muscle fibers, and branching was reductive (Fig. 1B,C, upper brackets).

Thus, in all three muscles, motor axons exhibited different growth patterns on slow versus fast myotubes. One way this might 
occur is if prespecified fast and slow motoneurons, with intrinsically different growth properties, were selectively targeted to the fast or slow region. Alternatively, some property of the muscle could impose the characteristic slow or fast branching pattern on any axons that entered the region. To determine whether motoneurons were selectively guided to innervate the fast and slow regions of their target or instead grew randomly into the muscle, we retrogradely labeled motoneurons projecting to fast and slow regions of the SART, AITIB, or IFIB muscles with the lipophilic dyes DiI (red) and DiAsp (green) and traced their axonal paths from the spinal cord to the muscle (Fig. 2).

\section{Axons to fast and slow muscle regions fasciculate separately in the nerve}

As shown for the SART muscle in Figure 2, axons projecting to the slow region (Fig. $2 A$, schematic, $B$ ) were labeled by injection of DiI into the distal slow region (Fig. 2B, black star). Axons projecting to the fast region of the same muscle were labeled by injection of DiAsp into the proximal fast region (Fig. 2C, black star). After dye injection, motoneuron cell bodies retrogradely labeled from each of these sites could be visualized within the sartorius motor pool in the spinal cord (Fig. 2B, C, black arrows). Furthermore, their axons could be followed throughout their trajectory in the limb (Fig. 2B,C), first as they grew within separate spinal nerves (arrowheads), then after the spinal nerves had converged in the crural plexus (short white arrows), and subsequently as the nerve trunk containing these axons physically diverged from the main crural trunk (long white arrows).

To analyze in more detail the behavior of axons at each of these points as well as to chart the distribution of their cell bodies within the spinal cord, we froze the cord and limb separately and sectioned them transversely.

After dye injection of fast and slow muscle regions in stage 35-36 embryos, cross-sections through the limb revealed a pattern of labeling suggestive of selective axonal fasciculation (Fig. 3). Cross-sections in Figure 3 show the position of axons labeled from the fast and slow regions of either the SART $(A-C)$, AITIB $(E-G)$, or IFIB $(I-K)$ muscles as they course through each of the specific points in the limb marked in Figure 2, $B$ and $C$ (arrows). Each muscle was injected in a different embryo. As shown in the top row of Figure 3, axons labeled from the fast (green) and slow (red) regions of either the SART $(A)$, AITIB $(E)$, or IFIB $(I)$ grew dispersed within the spinal nerves (nerves outlined by dashed white lines; section corresponds to the arrowheads in Fig. $2 B, C$ ) and intermixed with each other (areas of extensive mixing appear yellow) and with axons to unlabeled muscles (unlabeled axons within the outlined nerves appear dark). As the axons grew more distally and spinal nerves converged in the plexus region (for orientation, see Fig. $2 A$, schematic, $B, C$, short arrows), labeled axons began to occupy spatially discrete areas in the form- ing nerve plexus and to sort into muscle specific fascicles as described previously (Lance-Jones and Landmesser, 1981). Surprisingly, within the muscle-specific fascicle, a further sorting process occurred (Fig. 3B,F,J, cross-sections) that segregated fast(green) and slow-projecting (red) axons into separate but adjacent fascicles. This segregation of axons, established at the base of the limb, was maintained more distally as axons diverged from the main nerve trunk (Fig. $3 C, G, K$; for orientation, see also Fig. $2 B, C$, long arrows) and coursed toward their muscle targets. Although segregation of red-and green-labeled axons would be expected near the sites of dye injection in the muscle, the fact that sorting occurred at the base of the limb, long before axons reached the muscle, suggests that fast- and slow-projecting axons are distinct and respond differently to environmental cues. Similar results were seen after retrograde fast and slow injection in at least three additional limbs for each muscle (see Materials and Methods).

In addition to selectively fasciculating, fast- and slow-projecting axons also took different trajectories in the limb to reach the correct muscle fiber region of the target. For example, axons projecting to the SART (Fig. $3 A-C$ ) typically began to sort in the spinal nerves, where they gradually congregated in the anterior half of each spinal nerve (Fig. $3 A$ shows one such spinal nerve outline). As soon as two spinal nerves converged, SART axons moved toward each other; axons projecting to the slow region (red) congregated in the ventroanterior portion of the nerve, whereas those projecting to the fast region (green) became located slightly dorsal to but overlapping those projecting to the slow region (data not shown). Finally, slightly more distal in the plexus region, where all spinal nerves converged, SART axon sorting was often completed (Fig. $3 B$ ), with fast (green) and slow (red) axons occupying adjacent but mutually exclusive locations in the nerve trunk (outline). From this point, the SART-specific fascicle became more defined and then physically diverged from the main nerve trunk (Fig. $3 C$ ). The unlabeled axons in this trunk belong to the lateral femoral cutaneous nerve that diverges from the SART muscle nerve just distal to this point. In some cases, SART axons completed their sorting slightly more distally, after diverging from the main nerve trunk. They then traveled over 300 $\mu \mathrm{m}$ as separate but adjacent fascicles until they branched near the muscle to enter the appropriate SART muscle region. Similar results were found for the AITIB $(E-G)$ and the IFIB $(I-K)$.

Because fast and slow myotubes occur in anatomically separate regions of muscle, axons labeled from these regions must diverge from each other to selectively project to these different regions. For all three muscles, one such divergence occurs before the nerve enters the muscle and thus constitutes a decision point in which fast and slow axons respond differently to guidance cues. Figure $3 D$ shows this decision point in the SART nerve of one

\footnotetext{
Figure 2. Retrograde and orthograde dye injections used to label motoneurons. $A$, Schematic of basic injection paradigm. DiI and DiAsp were injected into different muscle regions (retrograde injections) or ventral roots (orthograde injection) to differentially label motoneurons. $B$, Montage of an intact stage 36 embryo after typical retrograde dye injection. DiI was injected into the distal slow SART region (black star) and retrogradely labeled motoneuron cell bodies in the spinal cord (black arrow). Notice that trajectories of DiI-labeled axons can be followed from the spinal cord to the muscle as they course through the spinal nerves (arrowhead), plexus (short white arrow), and muscle nerve, as it diverges from the main nerve trunk (long white arrow). C, DiAsp injection into the proximal fast region of the same muscle (black star). This injection labels a different population of neurons in the cord (black arrow) that send axons to the SART through the proximal branch of the SART nerve. Dyes DiI $(B)$ and DiAsp $(C)$ only label axons that come in direct contact with the dyes at the site of injection. Some myotubes labeled at the injection site extend further proximally $(B)$ or distally $(C)$ from this site and therefore are labeled with dye. This diff usion of dye along each labeled myotube, however, does not cause other axons outside the injection site to be labeled. White arrows and the arrowhead to the right in $C$ denote the same key areas marked in $B$. A-C, Anterior is to the left, proximal is up, and myotubes run vertically. Scale bars, $1 \mathrm{~mm}$.
} 

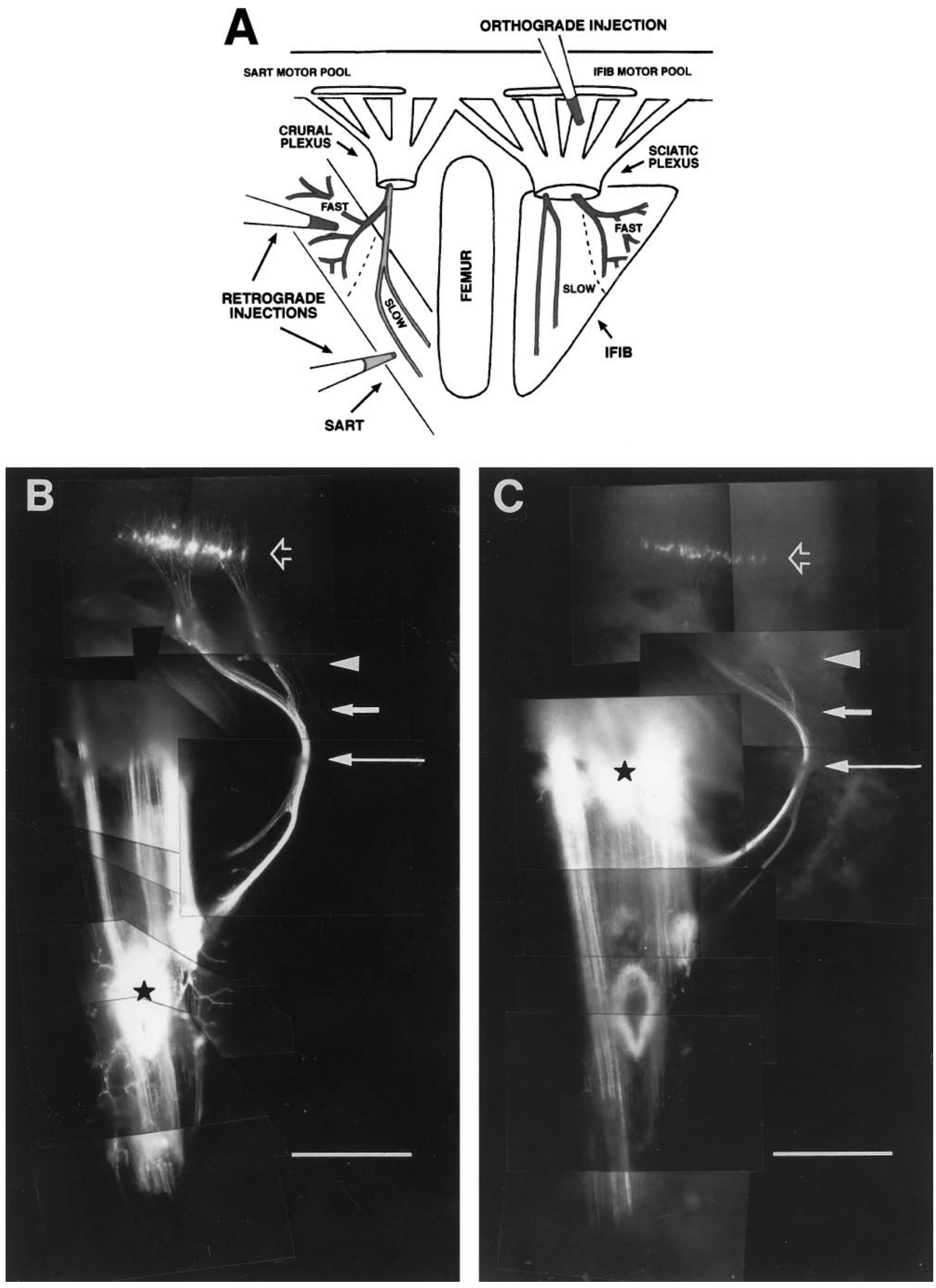

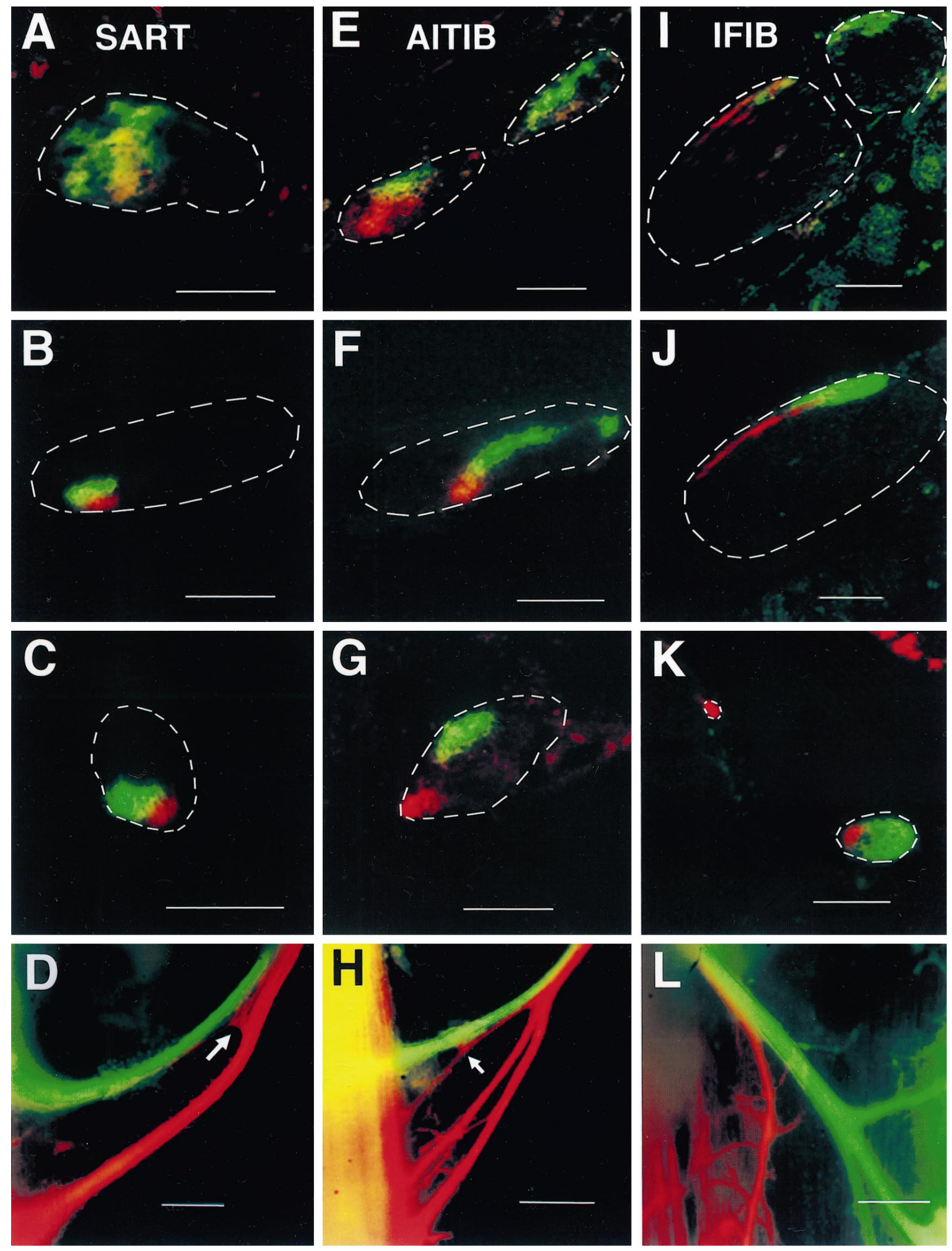

Figure 3. Fasciculation patterns of fast- and slow-projecting axons at different levels of the limb. Retrogradely labeled axons to fast and slow regions of three hindlimb muscles at stage 36 are shown: SART $(A-D, H), A I T I B(E-G)$, and IFIB $(I-L)$. Cross-sections through the limb show trajectories of retrogradely labeled fast- ( green $)$ and slow-projecting $($ red $)$ axons in the spinal nerves $(A, E, I)$, plexus region $(B, F, J)$, and more distally as muscle nerves diverged from main crural or sciatic nerve trunks $(C, G, K)$. Cross-sections through the spinal nerves $(A, E, I)$ showed that axons typically exited the spinal cord mixed together in the spinal nerves (extensive intermingling appears yellow) where they rapidly became more segregated. As the spinal nerves converged in the plexus region, axons sorted into muscle-specific fascicles that occupied stereotypical positions within the (Figure legend continues) 
embryo. In this whole mount (Fig. 3D), the SART nerve split into two branches before entering the muscle, with green-labeled fast axons entering the proximal branch and red-labeled slow axons entering the distal branch. Interestingly, some red-labeled axons turned at this bifurcation point (Fig. 3D, arrow) to enter the appropriate distal slow nerve branch. In another embryo (Fig. $3 H$ ), a few slow (red) SART axons remained in the proximal-most branch with the fast (green) axons and diverged closer to the muscle (arrow). Similar results were found for the AITIB (Fig. $3 G$, cross-section) in which fast and slow labeled axons diverged from each other even before they diverged from the femoralis nerve (outline). On the other hand, IFIB axons diverged at two decision regions. First, shortly after they sorted in the plexus, IFIB axons separated into two main nerves (Fig. $3 K$ ), one slow (red) nerve and one mixed (red and green) nerve. Once in the muscle (Fig. 3L, whole mount), the IFIB mixed nerve further diverged to enter the slow (red) and fast (green) labeled muscle regions.

In summary, axons to each of the three muscles studied initially sorted into fast and slow fascicles very proximally in the limb, long before they reached the muscle. These results strongly suggest that motoneurons projecting to fast and slow regions of the target are distinct and that from their initial ingrowth into the limb bud are able to segregate on the basis of their specificity for fast and slow muscle regions. In addition, the fact that axons to fast and slow regions took different trajectories through the limb and branched differently once within the muscle (as seen in Figs. 1, $3 L$ ) supports the hypothesis that fast- and slow-projecting axons are selectively guided to the appropriate muscle fiber region from the outset of innervation.

\section{Organization of fast- and slow-projecting motoneurons within the motor pool}

Motoneurons projecting to a single muscle are organized in coherent pools or clusters that occupy highly stereotypic positions within the ventrolateral spinal cord (Landmesser, 1978) (see also Fig. 2B,C, black arrows). Unknown is whether the cell bodies of fast- and slow-projecting motoneurons are also grouped in characteristic positions within each motor pool. If true, somal position might play a role in specifying motoneuron phenotype (Appel et al., 1995; Matise and Lance-Jones, 1996; Ericson et al., 1997) and could also be used to predict a priori which motoneurons would project to fast or slow muscle regions.

We therefore characterized the positions of fast- and slowprojecting motoneurons by backlabeling them with DiI or DiAsp from the separate fast and slow muscle regions of the SART, AITIB, and IFIB. Embryos were analyzed first as whole mounts (see Fig. 2B,C) in which the segmental distributions of DiI- and DiAsp-positive axons were estimated (Fig. 4G-I; see Materials and Methods) and later after cryostat sectioning when motoneuron cell bodies were counted and their positions within the lateral motor column were plotted by camera lucida-like tracings (Fig. 4D-F).

As seen in Figure $4 A-C$, motoneuron cell bodies in the ventrolateral cord were brightly labeled with dye after injection of fast and slow muscle regions. In this example (Fig. 4A-C), shown in black and white, a single DiI-labeled (slow) motoneuron (arrow) in the IFIB motor pool is seen at low magnification $(A)$ and at higher magnification $(B, C$, arrows) situated near two DiAsplabeled (fast) neurons (Fig. $4 C$, arrowheads). In all embryos analyzed, motoneurons were singly labeled by either DiI or DiAsp, indicating that a given cell projected only to the fast or slow regions of a muscle. Double-labeled cells were rarely seen $(<1 \%$ of cells). It is unclear whether these represent a small population of cells that branched into both fast and slow muscle regions or, more likely, were the result of imprecise dye injections.

Organization of the fast- and slow-projecting motoneurons in the motor pools differed for each muscle tested. In the SART pool, no preferential localization of fast- and slow-projecting motoneurons was observed on either the anterior-posterior (A$\mathrm{P})$, dorsal-ventral (D-V), or medial-lateral (M-L) axes (Fig. $4 D, G)$. For example, as seen in Figure $4 G$, fast (dark bars) and slow (light bars) labeled SART axons were equally likely to exit the cord from LS1 or LS2. Furthermore, the camera lucida drawings in Figure $4 D$ show that in the SART motor pool, fast (filled circles) and slow (open circles) retrogradely labeled motoneurons were extensively intermingled along the $\mathrm{D}-\mathrm{V}$ and $\mathrm{M}-\mathrm{L}$ axes. In contrast, for both the AITIB (Fig. 4H) and IFIB (Fig. $4 I$ ), motoneurons projecting to fast and slow muscle regions were organized to some extent along the A-P axis; the most rostral segments of each motor pool predominantly contained motoneurons projecting to the slow region (light bars), and the caudal segments contained motoneurons projecting to the fast region (dark bars). However, motoneurons in the central segments of these motor pools (LS2 for the AITIB; LS5 and LS6 for the IFIB) contained both fast- and slow-projecting motoneurons (see Fig. $4 H, I)$. In those segments of the AITIB and IFIB motor pools that contained both fast- and slow-projecting motoneurons, the organization of cell bodies was more variable. Overall, for neither motor pool was there a tight clustering of fast- and slow-projecting motoneurons along the $\mathrm{D}-\mathrm{V}$ or $\mathrm{M}-\mathrm{L}$ axes. For example, Figure $4 E$ shows the camera lucida from a typical AITIB motor pool (spinal cord level LS2). In this embryo, fast-

\section{$\leftarrow$}

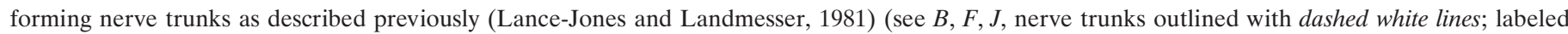

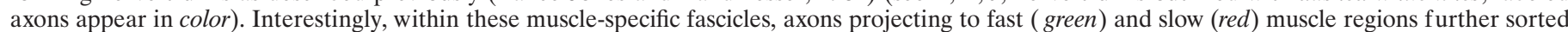

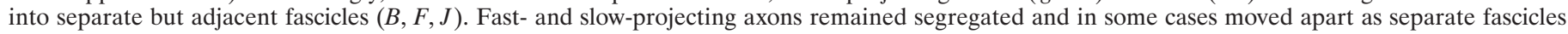

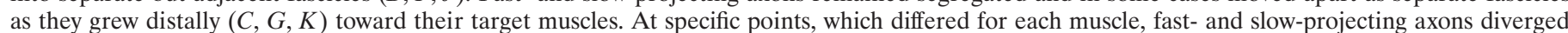

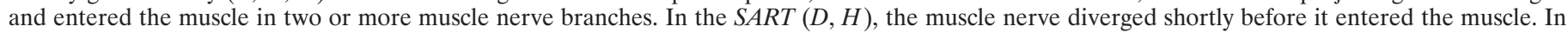

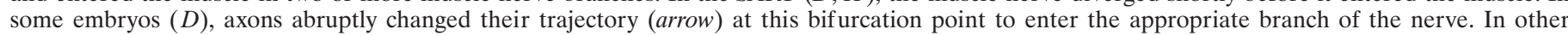

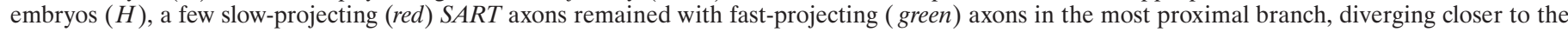

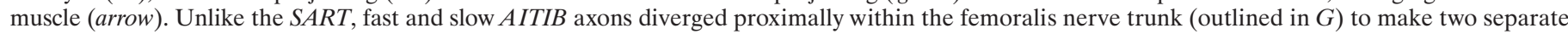

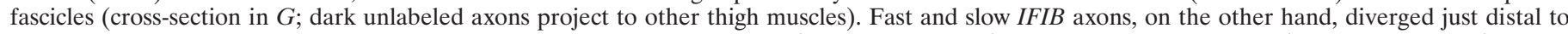

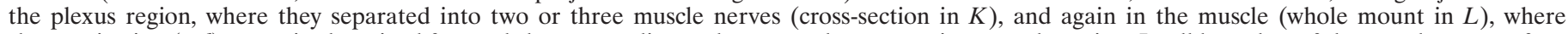

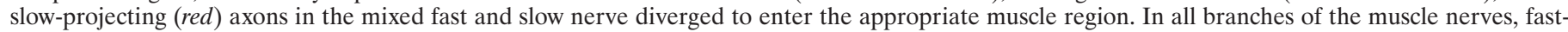

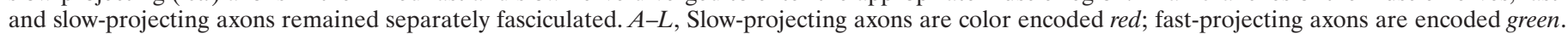

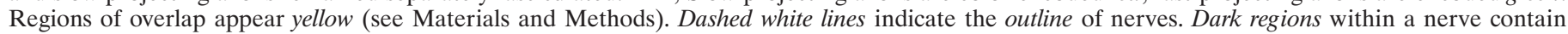

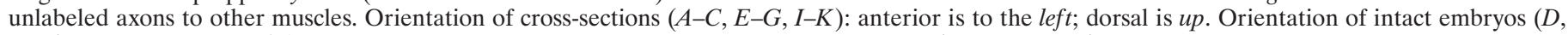
$H, L)$ : anterior is to the left; proximal is up. Scale bars: $A-C, E-F, I-K, 100 \mu \mathrm{m} ; D, H, J$ (whole mounts), $200 \mu \mathrm{m}$. 

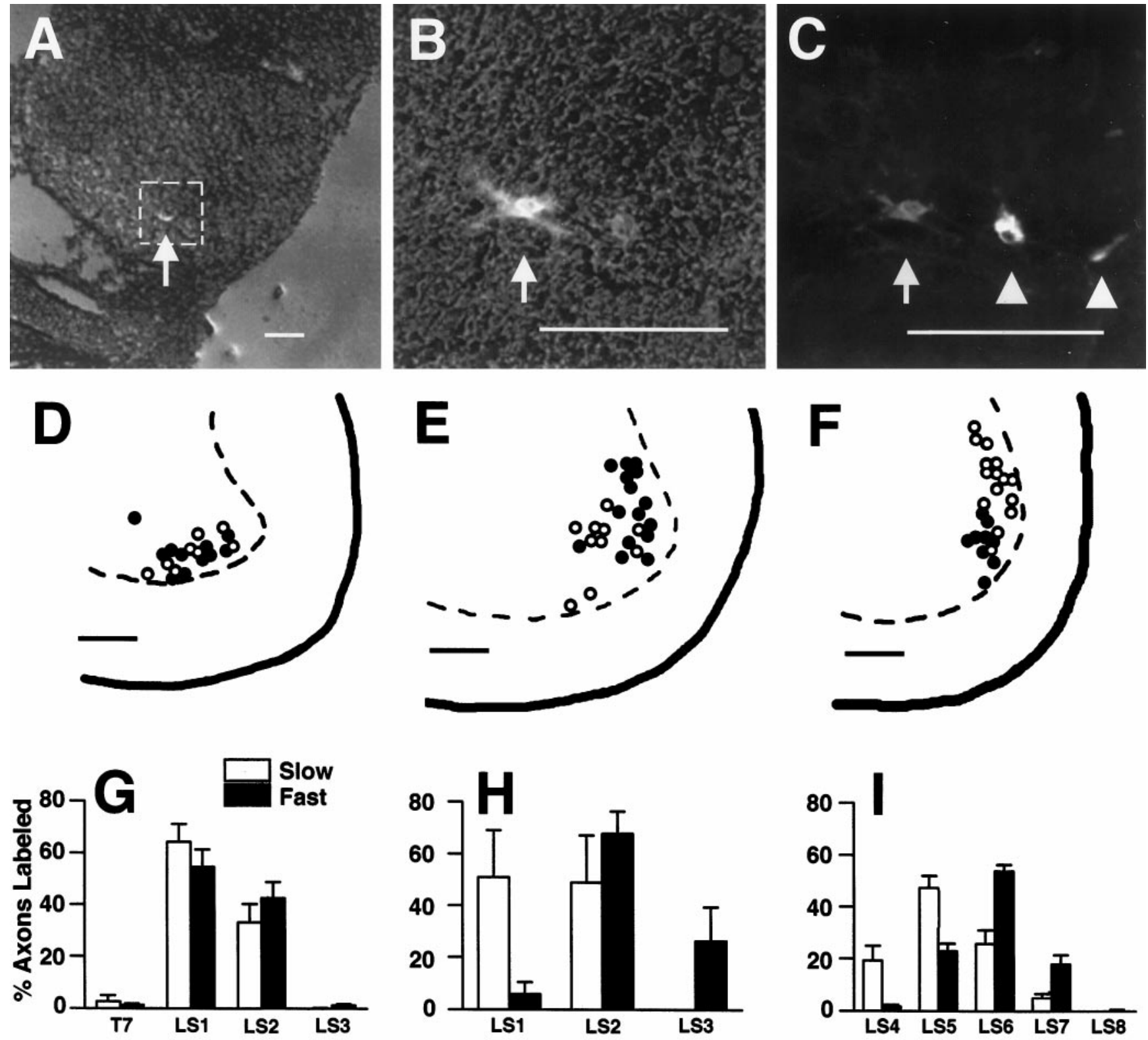

Figure 4. Distribution of fast- and slow-projecting motoneurons in the spinal cord at stage 36. $A-C$, Cryostat sections of lumbosacral spinal cord showing the location and morphology of dye-labeled motoneurons. A, Transverse section through LS5 showing one DiI-labeled cell (arrow) in the lateral motor column. The cord section was acquired at low magnification with both phase contrast and rhodamine epifluorescence to visualize the relative position of the cell body in the spinal cord. The box indicates the area magnified in $B$ and $C$. B. Higher magnification of the DiI-labeled cell in $A$ (arrow) visualized by phase contrast in the presence of rhodamine epifluorescence. $C$, Epifluorescence of the motoneurons in $B$. The DiI-labeled motoneuron appears gray (arrow) and resides close to two DiAsp-labeled motoneurons that appear white (arrowheads). Numerous processes emanating from the neuronal cell bodies are brightly labeled, and in some cells the nucleus is visible. The image was generated by color encoding and superimposition of images acquired under different epifluorescent filters and was printed in black and white (see Materials and Methods). $D-F$, Camera lucida-like tracings of dye-labeled motoneurons in the SART $(D)$, AITIB $(E)$, and IFIB $(F)$ motor pools in which the soma positions of motoneurons projecting to slow regions are shown as open circles and those to fast regions are filled circles (see Results for additional details). Orientation of camera lucidas: dorsal is up; medial is to the left. $G-I$, Bar graphs showing the segmental distribution of fast- and slow-projecting axons to the SART ( $G)$, AITIB $(H)$, and IFIB $(I)$. The $x$-axis: level of the spinal cord from the seventh thoracic (T7) through the eighth lumbosacral (LS8) segment that contained dye-labeled axons; the $y$-axis: proportion of total dye-labeled axons in each segment that projected to fast (dark bars) and slow (light bars) muscle regions \pm SE. $G$, Distribution of SART motoneurons. Approximately equal proportions of dye-labeled cells projected to fast (dark bars) and slow (light bars) regions of the SART from each cord segment (T7-LS3). The graph contains data from 22 dye-injected SART muscles. H, AITIB motoneuron distribution. Slow-projecting AITIB neurons (light bars) extended from $L S 1$ and $L S 2$ but not $L S 3$. In contrast, fast-projecting AITIB neurons (dark bars) resided mainly in $L S 2$ and $L S 3$ with a small number in $L S 1 ; n=3$ injected AITIB muscles. I, IFIB motoneuron distribution. Motoneurons to the slow IFIB (light bars) tended to reside rostrally in the motor pool ( $L S 4-L S 6$ ), whereas those to the fast IFIB (dark bars) were located more caudally ( $L S 5-L S 7)$. The graph contains data from 19 dye-injected IFIB muscles. Scale bars: $A-F, 100 \mu \mathrm{m} .$. 
and slow-projecting motoneurons are mostly intermingled along the $\mathrm{D}-\mathrm{V}$ and $\mathrm{M}-\mathrm{L}$ axes of the motor pool. In all AITIB pools, there was a slight tendency for fast-projecting motoneurons to be located dorsolateral to slow-projecting motoneurons. However, no clear boundary separated the groups of motoneurons into tight clusters. The only exception to this observation occurred in one IFIB motor pool (Fig. $4 F$ ) in which retrogradely labeled fast IFIB motoneurons ( filled circles) were located consistently ventral to slow IFIB motoneurons (open circles). This grouping was not typical of IFIB motor pools in two other pools documented by camera lucida. In fact, in most embryos, AITIB and IFIB fast and slow motoneurons were extensively intermingled and were clearly not clustered in the cord, in contrast to their axons in the plexus region. A minimum of two motor pools for each muscle was traced by camera lucida. The graphs in Figure $4 G-I$ combine data from 44 limbs (see legend for Fig. 4 and Materials and Methods).

In summary, although the axons of motoneurons projecting to fast and slow muscle regions selectively fasciculate in the limb, there is no tendency for their somas to be organized or clustered within the motor pool. Therefore cord position cannot be used to predict a priori which motoneurons will project to slow or fast regions nor can it explain the selective fasciculation of fast- and slow-projecting axons in the limb.

\section{Topography also plays a role in muscle innervation}

Analysis of the distribution of fast and slow motoneurons in the spinal cord (Fig. 4) showed that for two of the three muscles tested (AITIB and IFIB) motoneurons situated rostrally in the motor pool tended to innervate slow muscle regions, whereas those residing caudally innervated fast muscle regions (Fig. $4 H, I)$. Because the slow regions of each muscle are located anteriorly, whereas the fast regions are located posteriorly (for orientation, see also Fig. 1), we were interested to know whether this innervation pattern reflected a tendency for motoneurons to innervate muscles by anterior/posterior matching or fast/slow recognition. To understand the relationship of motoneuron soma position along the A-P axis and subsequent innervation of the target, we orthogradely labeled motoneurons with lipophilic dyes at the ventral roots of the spinal nerves (see Fig. $2 A$, schematic) and visualized their innervation patterns in muscle whole mounts at stage 35-36 (Fig. 5). Figure 5 shows the distribution of axons in the SART and IFIB muscles after injection of each of the relevant spinal nerves.

Orthograde labeling confirmed results from the retrograde labeling study of the SART and IFIB described above. For the SART, axons labeled from LS1 (Fig. $5 A$ ) or LS2 (Fig. 5B) projected to both the proximal and distal regions of the SART with no clear discrimination between fast and slow regions. On the other hand, in the IFIB, the most anterior motoneurons (LS4) primarily innervated the most anterior portion of the muscle (Fig. 5C), whereas the most posterior motoneurons (LS6 and LS7) primarily innervated the posterior portion (Fig. 5E,F) (compare with Fig. 1A). Importantly, LS5 (Fig. 5D) projected to both anterior (slow) and posterior (fast) regions of the IFIB. This innervation pattern indicates that IFIB motoneurons use anterior-posterior cues when innervating the muscle. However, because motoneurons from LS5 and LS6 projected to both fast and slow regions of the IFIB (see also Fig. 4I), it also suggests that the fast-slow matching that we observed cannot be explained simply as a consequence of anterior-posterior matching.

In summary then, the segmental organization of some motor pools (e.g., IFIB) corresponds grossly to the A-P area of the muscle innervated but does not correlate discretely with the innervation of fast or slow muscle fibers. The motoneurons within other motor pools (e.g., SART) exhibit no such A-P organization in the cord but, nevertheless, selectively innervate fast and slow muscle regions. These results suggest that although topography plays a role in target innervation, it is not a major determinant of the specific innervation of fast and slow muscle regions that we observed.

\section{The selective segregation of fast- and slow-projecting axons is not explained by specificity for separate muscle areas}

Rather than fast and slow recognition, the selective fasciculation of fast- and slow-projecting SART, AITIB, and IFIB axons could also be explained if axons selectively fasciculated with those projecting to the same discrete muscle area. To address this possibility, we injected the PITIB, an all-fast muscle located in the posterior thigh just dorsal to the IFIB. Axons enter this muscle on its anterior edge and project proximally and distally from this point as seen in the whole mount in Figure $6 \mathrm{~A}$. Although this muscle contains only fast muscle fibers, the proximal and distal regions of the muscle are separate compartments as shown by the fact that dye injection into these areas (Fig. $6 \mathrm{~A}$, arrow and arrowhead point to sites of injection) discretely labels separate branches of the muscle nerve as they enter the muscle (Fig. 6B, arrow and arrowheads in cross-section shown in black and white) as well as separate populations of myotubes (data not shown). Thus, using this muscle, we could test whether the selective fasciculation of fast- and slow-projecting axons was attributable to selective recognition of fast and slow motoneurons or recognition of axons projecting to topographically different muscle regions. We reasoned that if axons segregate by selective recognition of fast and slow identity, then PITIB axons, which are all-fast motoneurons, should not sort. On the other hand, if axon sorting is based on specificity of axons for discrete muscle regions, then PITIB axons, which project to the discrete proximal and distal compartments of this muscle, should sort.

Retrograde dye labeling of PITIB axons showed that at all levels of the limb, axons projecting to the proximal (red) and distal (green) regions of the PITIB were significantly more intermixed (Fig. 7) than were axons projecting to the fast and slow regions of the SART, AITIB, and IFIB (see Fig. 3). Most proximally in the limb (Fig. $7 A$, cross-section), PITIB axons were extensively intermixed within the spinal nerves (nerve outlined in white). Soon after, as the spinal nerves converged in the plexus region (Fig. 7B), axons to the proximal (red) and distal (green) muscle compartments could be seen separating from each other as though sorting along the dorsoanterior edge of the forming sciatic nerve (Fig. 7B, yellow indicates region of mixing; nerve trunk outlined in white). However, as the axons grew more distally and PITIB axons grouped in a muscle-specific fascicle, axons to proximal (red) and distal (green) regions again began to mix together. Mixing was complete by the time the PITIB muscle nerve separated from the sciatic and grew toward the muscle (Fig. $7 C$, axon intermixing shown by yellow; muscle nerve outlined in white). In some cases, PITIB axons appeared completely mixed together in the muscle nerve (Fig. $7 C$, yellow). In other cases, they appeared partly sorted (Fig. 7D, arrow shows remaining area of overlap) but became more overlapped as they grew distally toward the target (data not shown). Mechanisms underlying this variability in PITIB sorting are unclear. It is possible that axons 


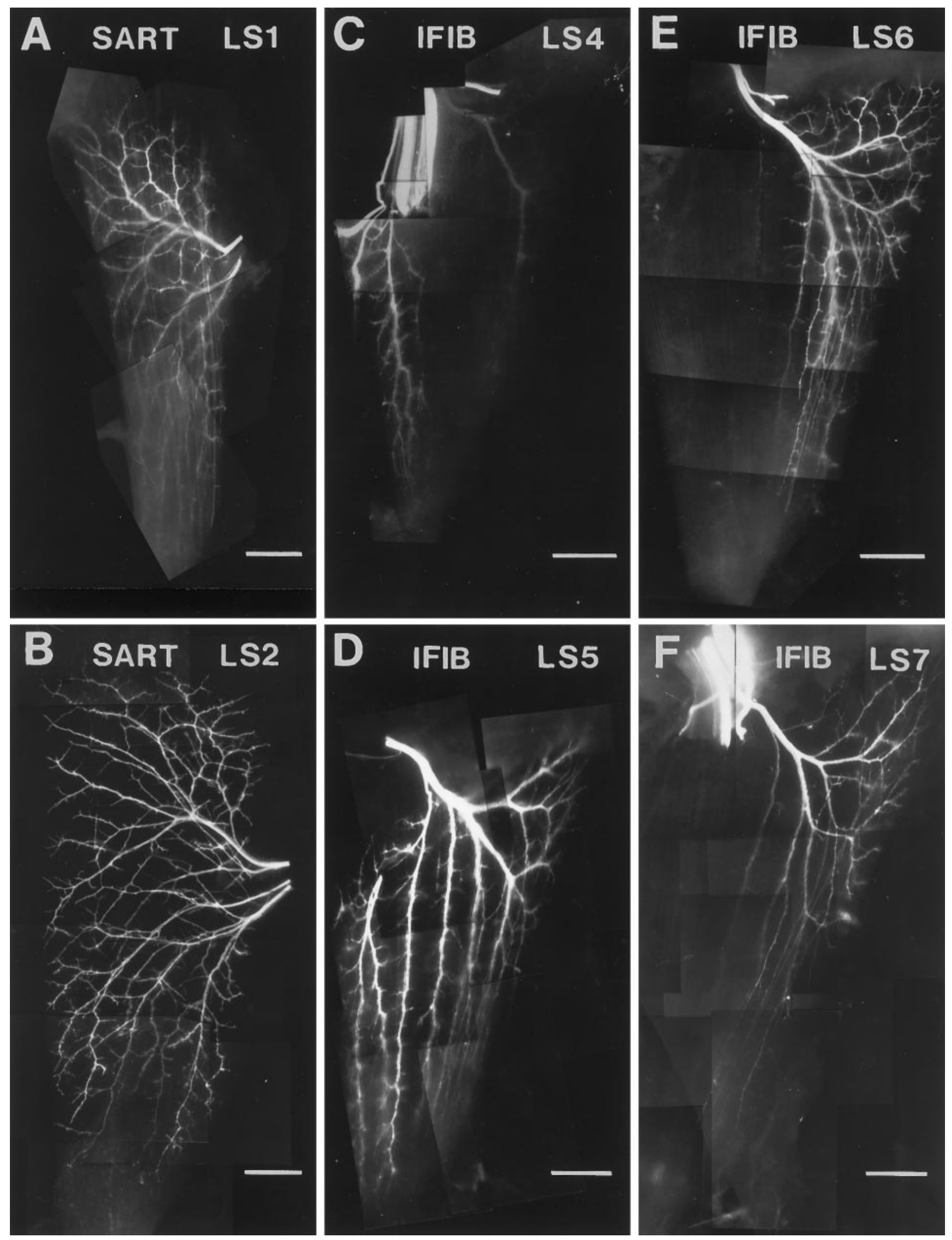

Figure 5. Muscle whole mounts after ventral root DiI injections at stage 35-36. A, B, SART whole mounts. Orthograde DiI injection into the ventral roots of $L S 1(A)$ or $L S 2(B)$ resulted in labeling of motor axons in both slow and fast regions of the $S A R T$ (compare with Fig. $1 B$ ). The difference in extent of branching between $A$ and $B$ reflects the younger embryonic stage of $A$. $C-F, I F I B$ whole mounts. Injection of DiI into the ventral root of $L S 4$ $(C)$ labeled axons projecting predominantly to the anterior slow region of the IFIB. LS5 injection $(D)$ labeled axons to the whole $I F I B$, including both slow and fast muscle regions. LS6 injection $(E)$ predominantly labeled axons in the posterior IFIB but contained both fast- and slow-projecting axons. In contrast, $L S 7$ injection predominantly labeled axons in the fast $I F I B$ region $(F)$. In all panels, anterior is to the left, proximal is $u p$, and myotubes run vertically. Truncated sciatic nerves are brightly labeled in $C$ and $F$ (top left). Scale bars, $500 \mu \mathrm{m}$. 

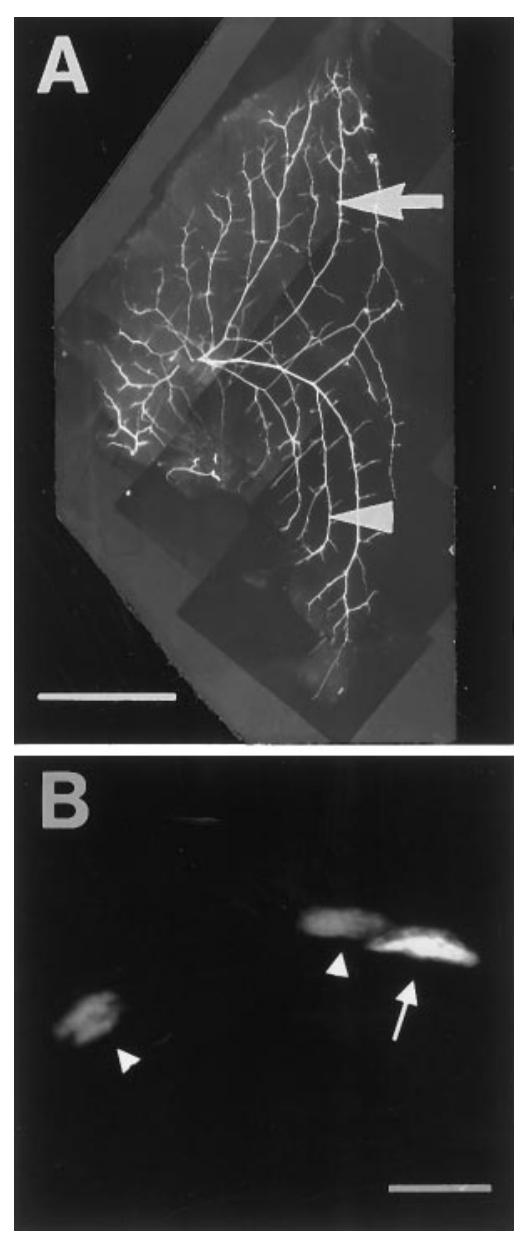

Figure 6. The all-fast PITIB is a compartmentalized muscle. A, PITIB muscle whole mount stained with anti-neurofilament antibody to show intramuscular nerve branching. The PITIB nerve enters the muscle at its anterior edge (left) and branches immediately to innervate the proximal (arrow) and distal (arrowhead) compartments of the muscle. Muscle fibers run vertically. DiI injection into the proximal (arrow) and DiAsp injection into the distal (arrowhead) muscle regions labeled different branches of the PITIB muscle nerve as seen in cross-section in $B$. $B$, Transverse section through the limb shows PITIB muscle nerves labeled by dye injection. The PITIB nerve shown as white (arrow) was labeled from dye injection into the proximal region (arrow in $A$ ). Nerves shown as gray (arrowheads) were labeled from the distal region (arrowhead in $A$ ). Scale bars: $A, 1 \mathrm{~mm} ; B, 50 \mu \mathrm{m}$.

projecting to different muscle compartments undergo selective sorting to a more limited extent than do fast and slow axons. Alternatively, the variability could be an artifact of dye labeling in cases in which DiAsp did not brightly label axons all the way back to the spinal cord. In this case, the resulting imbalance of dye intensities when fluorescent images were superimposed could result in the inappropriate appearance of less axon mixing more proximally than distally in the limb, like that seen in the embryo in Figure $7 D$.

In summary, despite some possibility of topographic-based sorting (e.g., Fig. 7D), the extensive intermingling of PITIB axons that project to topographically discrete muscle compartments was in striking contrast to the segregation of axons projecting to the fast and slow regions of the three mixed muscles studied. This result thus supports the hypothesis that selective fasciculation of axons to the fast and slow SART, AITIB, and IFIB is based in large part on the differential recognition of fast and slow motoneurons rather than on specificity to innervate a particular muscle region or compartment.

\section{The pattern of fasciculation observed is not established by selective axon retraction or cell death}

Because sorting of fast- and slow-projecting axons was seen proximally, at the base of the limb, it is likely to reflect the pattern of axon growth into the limb. However, because the observations thus far were made on older embryos near the end of the period of naturally occurring motoneuron cell death, selective axon retraction or cell death may have contributed to the apparent segregation of axons. To address this, we injected a series of embryos at stage $30(n=26)$, before the period of naturally occurring motoneuron cell death and any synaptic rearrangements that might subsequently occur (Hamburger, 1975; for review, see Oppenheim, 1991). It is also the stage when motor axons first enter the muscles (Lance-Jones and Landmesser, 1981; Tosney and Landmesser, 1985; Dahm and Landmesser, 1988) and is the first stage in which muscles are fully cleaved from the embryonic muscle mass (Romer, 1927; Schroeter and Tosney, 1991) and available for differential fast or slow injection.

Both orthograde and retrograde dye injections at stages 29-31 revealed the same pattern of axon sorting and motor pool organization seen at older stages. As shown in Figure $8 A$, motoneurons backlabeled from the fast ( green) and slow (red) regions of a stage 31 SART appeared mixed together in the spinal nerves (Fig. $8 A$, spinal nerve outlined in white; yellow indicates intermixing of labeled axons). As the spinal nerves converged in the plexus (Fig. $8 B$ ), SART axons sorted into separate but adjacent red (slow) and green (fast) fascicles in the forming nerve trunk (outline) and remained segregated more distally in the SART muscle nerve (Fig. 8C, outline) as it grew toward the target. The light and dark bars in Figure $8 D$ show that these fast and slow labeled axons were distributed equally in the spinal nerve segments LS1 and LS2, similar to the distributions at later stages (compare Figs. 8D and $4 G)$.

Similarly, axons to the IFIB at stage 30 (Fig. $8 E-G$, crosssections) were initially mixed in the spinal nerves (Fig. $8 E$, two spinal nerves outlined in white; area of overlap in LS5 marked by an arrow) but became more localized and segregated at the base of the limb (Fig. $8 F$ ) as axons projecting to the slow region (red) separated from those to the fast region (green) (sciatic nerve outlined in white). As the axons grew closer to the muscle (Fig. $8 G)$, they diverged from the sciatic as three individual muscle nerves, two slow (red) nerves seen to the left (outlined in white) and a larger fast (green) nerve to the right (outlined in white). The differential distribution of fast- and slow-projecting IFIB motoneurons along the A-P axis seen in older embryos was also evident at stage 30 (Fig. $8 H$ ). Axons projecting to the slow IFIB (light bars) exited the anterior-most spinal nerves (LS4-LS6), whereas those projecting to the fast IFIB (dark bars) tended to be in LS5-LS7 (compare Figs. $8 H$ and $4 I$ ).

These results indicate that axon sorting in the limb as well as the topographic innervation of selected muscles occurs before motoneuron cell death and synaptic rearrangement and therefore likely represents the trajectories taken by axons as they first grew into the limb.

\section{Role of electrical activity in axon sorting and target selection}

It has been proposed that electrical activity could play a role in axon guidance and target selection (Goodman and Shatz, 1993). 

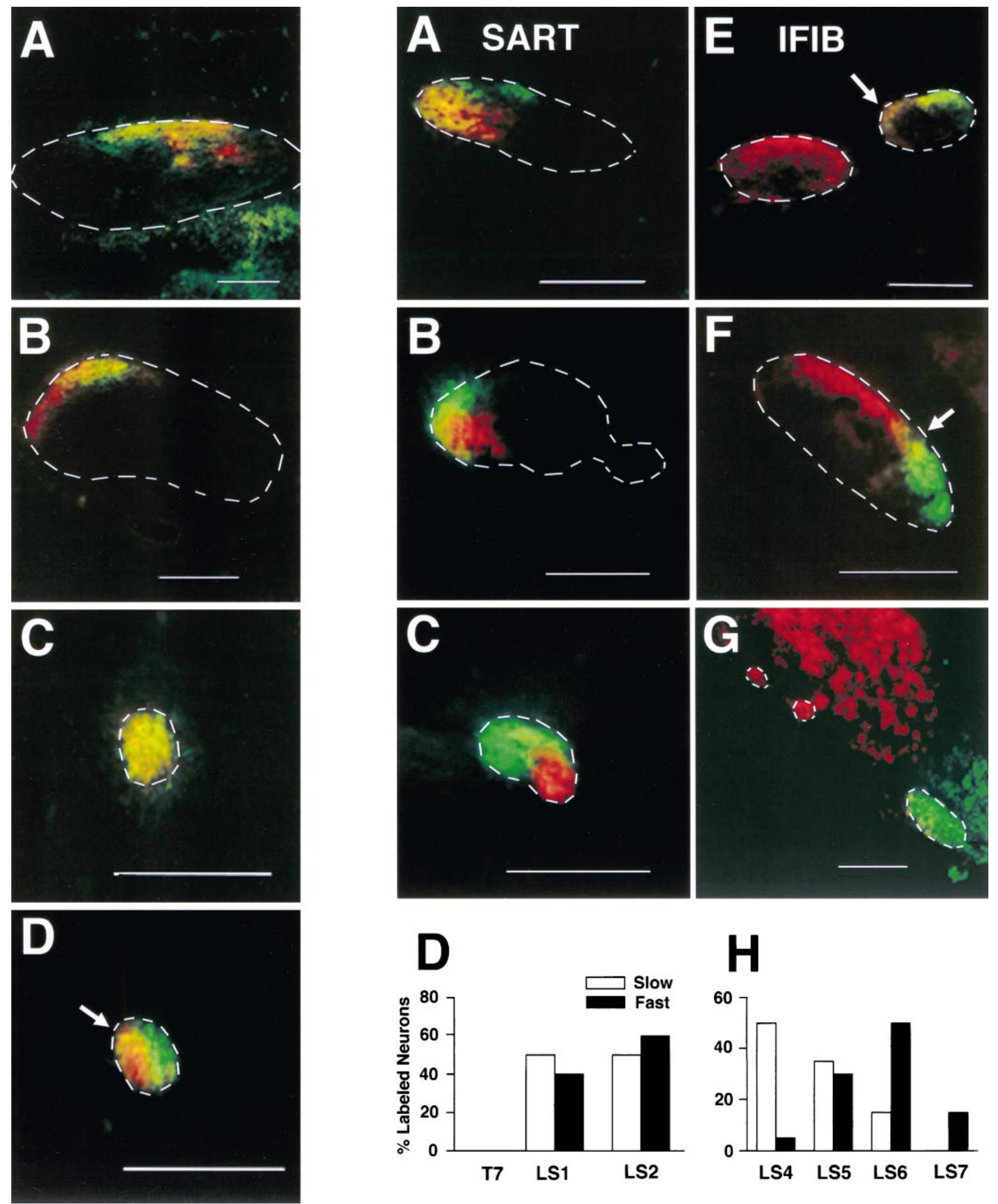

Figure 7. Left. PITIB axons innervating different muscle compartments do not separately fasciculate in the nerve. $A-C$, Retrogradely labeled PITIB axons from a single embryo shown in cross-section at different levels of the limb. A, Axons projecting to the proximal (red) and distal (green) compartments of the PITIB exit the spinal cord mixed together and course along the dorsal edge of the spinal nerve. $B$, In the plexus, PITIB axons group in the dorsoanterior corner of the sciatic nerve trunk (nerve trunk outlined in white) where they appear partially to sort. $C$, At the point at which the PITIB nerve separates from the sciatic, PITIB axons are completely mixed together (yellow; nerve outlined in white). D, In a different embryo, PITIB axons remained partially sorted in the proximal portion of the muscle nerve (arrow indicates area of axon mixing) but (Figure legend continues) 
Patterned electrical activity has been shown to be required for the normal segregation of lateral geniculate axons into ocular dominance columns in the visual cortex (for review, see Goodman and Shatz, 1993). Furthermore, previous studies have shown that the anterior and posterior regions of the IFIB have different patterns of activation in an in vitro spinal cord-hindlimb preparation at stage 36 (Landmesser and O'Donovan, 1984a,b; Vogel, 1987). Therefore, we were interested to know whether activity could play a determining role in the segregation of axons into fast and slow fascicles within the muscle nerve or the subsequent innervation of discrete regions of the muscle target. To address this, we recorded EMGs from the fast and slow regions of the SART, AITIB, and IFIB muscles. Muscle activity, as recorded by EMGs in the chick, has been shown to mimic the activity of the innervating motoneurons (Landmesser and O'Donovan, 1984a,b; O'Donovan, 1989). Therefore, by measuring EMGs, we were able to determine the activity pattern of the motoneurons projecting to fast and slow muscle regions.

As described previously (Landmesser and O'Donovan, 1984a; O’Donovan, 1989; Ho and O'Donovan, 1993; Sernagor et al., 1995; Sholomenko and O'Donovan, 1995), stimulation of descending input with a single electrical shock to the thoracic cord activates a central pattern generator in the lumbosacral cord, setting off a series of highly stereotyped motoneuron bursts (or cycles) characteristic for each muscle. Figure $9 A$ shows the EMG to one such muscle, the AITIB, after a single stimulus to the cord. Interestingly, in the AITIB, we found that fast and slow muscle regions have distinctly different bursting patterns. This is evident in both the EMGs (on the left) and the histograms (on the right) that plot the probability of AITIB motoneuron activity at different points in the bursting cycle. For orientation, one cycle in the EMG is marked by a bracket; an arrow marks cycle onset. In the slow region of the AITIB (Fig. 9A, top trace), bursting was flexor-like; axons were active before the onset of the cycle, then were quiescent for $\sim 400$ msec (thick bar) after the synchronous response that typically marks cycle onset (arrow), and resumed bursting after this inhibitory period (see also adjacent histogram). On the other hand, the fast region (Fig. $9 \mathrm{~A}$, bottom trace) was characterized by a more extensor-like pattern in which axons burst predominantly at the onset of each cycle with no clear inhibitory period (arrow marks cycle onset; see also adjacent histogram).

Consistent with earlier reports (Landmesser and O'Donovan, 1984a,b; Vogel and Landmesser, 1987), the fast and slow regions of the IFIB also exhibited different patterns of activation after a single stimulus (Fig. 9B). In the slow region (Fig. 9B, top trace and adjacent histogram), the IFIB was activated very synchronously at the onset of a cycle (arrow; one cycle is marked by a bracket for orientation). In contrast, in the fast region (Fig. 9B, bottom trace), this initial burst of activity was followed by a period of quiescence and then more activity (asterisk). As seen in the EMGs, this second period of activity occurred reproducibly in the fast region (Fig. 9B, bottom trace, asterisk) but rarely in the slow region (Fig. $9 B$, top trace). Thus motoneurons that project to the fast and slow regions of both the AITIB and IFIB not only project differently in the periphery but are also activated differently by the central pattern generator. These results suggest that differential activity, like that seen in the fast and slow AITIB (Fig. 9A) or IFIB (Fig. $9 B$ ), could play a role in axon sorting or the subsequent restriction of fast and slow axons to the appropriate muscle regions. However, fast and slow regions of the SART do not exhibit differences in their pattern of activity (Fig. 9C), suggesting that such differential activity is not required for appropriate sorting.

\section{DISCUSSION \\ Targeting of motoneurons to fast and slow muscle regions}

Mechanisms underlying the matching of motoneuron type with muscle fiber type may involve the specific recognition of fast and

became more mixed as they grew distally toward the muscle. This section in $D$ was taken from the limb position equivalent to that in $C$. In all panels, red labels axons projecting to the proximal PITIB, green labels axons to the distal PITIB, and yellow indicates areas of axon mixing. Fifteen PITIB-injected limbs were analyzed, and all showed much more extensive mixing than was seen in mixed fast and slow muscles. Orientation of all panels: anterior is to the left; dorsal is up. Dashed lines outline nerve boundaries. Unlabeled axons (dark) within the outlined nerves project to other hindlimb muscles. Scale bars, $100 \mu \mathrm{m}$.

Figure 8. Right. Axon sorting and fast and slow targeting occur before the period of motoneuron cell death and are similar to that seen at later stages. $A-C, E-G$, Sequence of limb cross-sections showing the position of motor axons backlabeled from the fast ( green) and slow (red) regions of the $S A R T$ at stage $31(A-C)$ and the IFIB at stage $30(E-G) . D, H$, Cord distribution of these motoneurons in the $S A R T(D)$ and $I F I B(H)$ motor pools. $A-D$, Data are from a single embryo as are data in $E-H$. A, Fast (green) and slow (red) labeled SART axons exited the spinal cord mixed together (yellow) in the spinal nerves and quickly began to sort and move toward the anterior half of the spinal nerve (nerve outlined in white). Unlabeled axons (dark) in the spinal nerves project to other muscles in the anterior thigh. $B$, In the plexus region, $S A R T$ axon sorting was completed, and fast-projecting axons ( green) consolidated dorsal and anterior to the slow-projecting axons (red), a distribution that was also seen at later stages (compare with Fig. $3 B$ ). $C$, Fast(green) and slow-projecting (red) $S A R T$ neurons remained segregated in the muscle nerve as they coursed through the limb. In the limb, the fast and slow fascicles rotated about each other such that the fast fascicle became located primarily anterior to the slow fascicle by the time it reached the $S A R T$ muscle. Orientation of $A-C$ : anterior is left; dorsal is up. $D$, Graph of motoneurons from the same embryo in $A-C$ shows the proportion of labeled axons projecting to the fast and slow SART ( $y$-axis) from each spinal segment ( $x$-axis). As shown by the light and dark bars, motoneurons to the fast (dark bars) and slow (light bars) SART exited the cord in both LS1 and LS2 in proportions similar to those at later stages (compare with Fig. 4G). Because motoneuron cell bodies at young stages were small and hard to distinguish from labeled dendrites, the percent of labeled neurons in the graph was visually estimated from the distribution of labeled axons in the spinal nerves of the intact embryo (see Materials and Methods). Results were similar in two other embryos and were confirmed by orthograde injections of LS1 and LS2 at this stage (data not shown). E, IFIB axons in two spinal nerves, LS4 (left) and LS5 (right), from a stage 30 embryo are shown. LS4 contained axons projecting only to the slow IFIB (red), whereas LS5 contained axons to both slow (red) and fast ( green) IFIB regions. Note that axons to the fast and slow IFIB are mixed together in LS5, especially in the anterior half of the spinal nerve (arrow). F, In the plexus, IFIB axons coursed along the dorsal edge of the developing sciatic nerve trunk (outlined in white) and slow-projecting (red) axons sorted from fast ( green) axons. The arrow points to a few remaining unsorted axons. $G$, IFIB axons separated into three distinct nerves soon after they sorted in the plexus. Dashed lines outline the IFIB nerves projecting to the slow (red) or fast ( green) regions of the muscle. $H$, Distribution of labeled $I F I B$ axons in all spinal nerves of the same embryo shown in $E-G$. Note the unequal distribution of fast- and slow-projecting motoneurons from the spinal cord; slow-projecting axons (light bars) originated predominantly from anterior segments (LS4-LS6), whereas fastprojecting axons (dark bars) resided mostly in posterior segments (LS5-LS7). Distribution of motoneurons to the fast and slow IFIB was the same before and after cell death (compare Figs. $8 H$ and $4 I$ ). Axons to slow muscle regions are labeled red; axons to fast muscle regions are labeled green. Yellow indicates areas of axon mixing. Dark areas within a nerve contain axons projecting to unlabeled muscles. Dashed lines outline the nerves. Orientation of cross-sections: anterior is to the left; dorsal is up. Scale bars, $100 \mu \mathrm{m}$. 
Figure 9. EMG activation patterns recorded simultaneously from fast and slow muscle regions in three stage 36 embryos. In an in vitro spinal cord-hindlimb preparation, a single electrical stimulus to the thoracic cord elicits a series of motoneuron bursts or cycles (see Results for more detail). The EMGs for fast and slow muscle regions of the $A I T I B, I F I B$, and $S A R T$ are shown on the left. The extent of one cycle in each EMG is marked by a bracket, arrows mark cycle onset, and the thick horizontal bar in $A$ indicates an inhibitory period just after cycle onset that is characteristic for this muscle. All EMGs have the same time base shown by the calibration bar at the bottom of the figure. The histograms to the right, which combine data from a number of cycles, show the probability of a pool being active at different phases of the step cycle. In the histograms, the onset of the cycle is indicated by the 0 time point in the graph. The bracket marks a cycle; the thick horizontal bar indicates the inhibitory period. $A$, The fast and slow regions of the $A I T I B$ were differentially activated by the central pattern generator. The slow region (top trace) was activated in a flexor-like pattern. It was active before cycle onset, became quiescent for $\sim 400 \mathrm{msec}$ (thick horizontal bar) after cycle onset (arrow), and then resumed bursting. In contrast, the fast region (bottom trace) was extensorlike and burst for a shorter period beginning at the onset of a cycle (arrow). Histograms (right) for each region show the probability of activation at any time in the cycle (0 marks cycle onset). $B$, The fast and slow regions of the $I F I B$ were also differentially activated. Both regions exhibited a brief synchronous activation at the onset of each cycle (arrows). In addition, the fast region (bottom trace) but not the slow region (top trace) was frequently active again (asterisk) after a period of quiescence. The adjacent histograms show graphically the probability of activation during any point in the cycle $(0$ marks cycle onset). $C$, In striking contrast to the $A I T I B$ and $I F I B$, there was no detectable difference between the activation patterns of the fast and slow SART regions. Arrows in EMGs mark cycle onset. Histograms (right) show the probability of activation of the $S A R T$ muscle during a cycle.
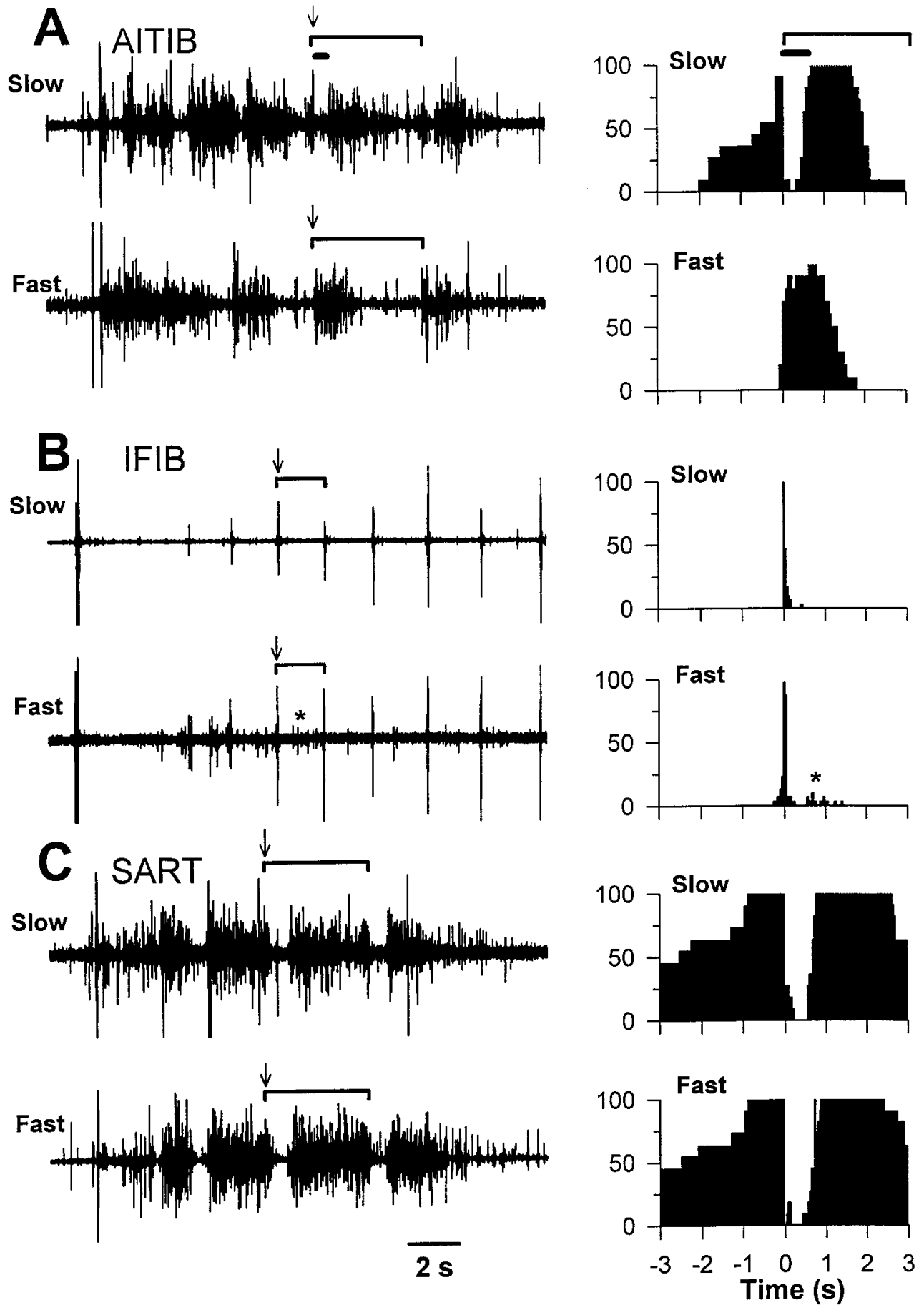

slow muscle fibers by molecularly distinct fast and slow motoneurons early in development when axons first enter the target. The present findings strongly support this hypothesis because chick motor axons projecting to the fast or slow regions of a single muscle were shown to fasciculate separately as they pathfind through the limb and once at the target to grow directly to their appropriate muscle fiber-containing region. Selective fasciculation was similar both before and after the motoneuron cell death period, showing that the observed fasciculation was not caused by selective neuronal cell death or synaptic rearrangements within the muscle. We also found that the position of motoneuron somas in the spinal cord was not clearly correlated with fast and slow specificity or subsequent axon fasciculation. These results are consistent with previous reports of selective innervation both in the chick, showing that embryonic motoneurons selectively innervate foreign muscles containing the muscle fiber type characteristic of their normal target (Rafuse et al., 1996; for adult chick, see also Feng et al., 1965; Hnik et al., 1967), and in the mammal, documenting that neonatal motor units are composed predominantly of a single muscle fiber type (for mouse, see Fladby and Jansen, 1988, 1990; for rat, see Thompson et al., 1984, 1987, 1990; for rabbit, see Gordon and Van Essen, 1985; Soha et al., 1987; Cramer and Van Essen, 1995). 


\section{Determinants of selective fasciculation and pathfinding}

What are the players involved in imparting fast or slow specificity and directional guidance to ingrowing chick motor axons? Although the identity of these cues remains unknown, previous studies suggest that chick motoneurons use both contactmediated and diffusible cues to find their targets (for review, see Landmesser, 1992). The present study further indicates that motoneurons may express a variety of different molecules reflecting multiple levels of specificity and may use these molecules to fasciculate selectively with axons sharing similar identities.

Four levels of specificity were observed: specificity for (1) muscle target, (2) fast or slow primary myotubes, (3) muscle compartment, and (4) A-P muscle areas. Axons sharing all four identities sorted the most completely into separate fascicles in the plexus region (e.g., IFIB). Others such as PITIB axons, sharing only target and compartment specificity, sorted to a lesser extent, and mixing between axons increased as they grew closer to the muscle. In addition, in two of the four muscles tested, orthogradely labeled motoneurons projected preferentially to the anterior or posterior portions of the target corresponding to their rostral or caudal somal positions in the motor pool, respectively. This topographic innervation did not correspond to the spatial distribution of fast and slow muscle regions and therefore may represent another level of target specificity. Some mammalian muscles are also topographically innervated (Browne, 1950; Swett et al., 1970; English and Weeks, 1984; Weeks and English, 1985; Donahue and English, 1987; Laskowski and Sanes, 1987; BaliceGordon and Thompson, 1988; Laskowski and High, 1989), supporting a role for A-P cues in muscle innervation.

The molecules responsible for selective fasciculation have yet to be identified. It has been shown that axons can fasciculate as a result of either attractive or repulsive molecules. Classes of molecules shown to be involved in fasciculation and pathfinding include the cell adhesion molecules, Eph kinases and their ligands, the semaphorins/collapsins, and the chemoattractants/repellents such as netrin and HGF/SF (for review, see Goodman, 1996). Each of these classes has been postulated to play a role in motor axon targeting in vertebrate or invertebrate systems (e.g., Donoghue et al., 1996; Ebens et al., 1996; Mitchell et al., 1996; Wang and Anderson, 1997; but see Kitsukawa et al., 1997). However, for none of these classes has the distribution of ligands and receptors been characterized on a sufficiently detailed level to predict whether they are likely to be involved in the fast/slow fasciculation and targeting observed in the present study, nor have experimental perturbations been performed to test these possibilities.

In addition to selective fasciculation, chick motoneurons clearly make different pathfinding choices in the limb and take specific trajectories to their target and appropriate target region. Because choices often involve the divergence of a subset of axons from the main nerve, fasciculation must be regulated carefully to allow defasciculation at key choice points. In Drosophila, two phosphatases, DLAR and DPTP, play an important role in motor axon defasciculation (Desai et al., 1996, 1997; Krueger et al., 1996) as does BEAT, a molecule secreted by a subset of motor growth cones (Fambrough and Goodman, 1996). Although homologous molecules have not been described in the chick, axon defasciculation seems to be enhanced by high levels of polyscialic acid (PSA) on a neural cell adhesion molecule (Landmesser et al., 1988, 1990). PSA is expressed more highly on axons projecting to fast as opposed to slow muscle regions and seems to regulate the pattern of intramuscular nerve branching (Landmesser et al., 1988, 1990). Further- more, early enzymatic removal of PSA results in motoneuron pathfinding errors and enhanced fasciculation in both the nerve and the muscle (Landmesser et al., 1988, 1990; Tang et al., 1992, 1994). A direct test of the role of PSA in the selective fasciculation of fast and slow motor axons or in their subsequent selection of fast and slow muscle regions has yet to be performed.

\section{Activity patterns in fast and slow muscle regions}

That fast- and slow-projecting motoneurons exist as distinct subclasses is also supported by their different patterns of activation in several motoneuron pools. The different activity patterns recorded from the fast and slow regions of the AITIB and IFIB indicate that these fast and slow motoneurons are differentially activated by the central pattern generator (CPG) (O'Donovan, 1989), presumably via differential innervation by local interneurons (O’Donovan, 1989; Ho and O'Donovan, 1993; Sernagor et al., 1995). Because fast- and slow-projecting motoneurons were mixed within the motor pool, selective connectivity suggests the recognition of specific molecular cues on the motoneuron cell bodies/dendrites by local interneurons. Additionally, differences in intrinsic membrane properties (transmitter receptors, channels, etc.) between fast and slow motoneurons may have contributed to these differences in activation (Sernagor et al., 1995).

The resulting patterned activity generated by the CPG, however, does not seem to play a determining role in the selective fasciculation of motor axons in the limb or in their subsequent targeting to fast and slow regions. This is supported by the observation that although motoneurons to each of the three muscles tested underwent selective fasciculation and selective targeting, different patterns of activity were shown to occur in the fast and slow regions of only the AITIB and IFIB (see also Landmesser and O'Donovan, 1984a; Vogel, 1987) but not the SART. Nevertheless, electrical activity may still be required for normal projections to develop. We have recently found that chick lumbosacral motor pools exhibit spontaneous patterned bursts of activity as they grow to their targets (L. Milner, M. Usiak, and L. Landmesser, unpublished observations). Because the expressions of several adhesion/guidance molecules are activity dependent (Landmesser et al., 1990; Fredette et al., 1993; Fields and Itoh, 1996; Rafuse and Landmesser, 1996), electrical activity could be required for proper fasciculation/pathfinding by regulating the expression of such molecules. Pharmacological studies altering motor activity are underway to test this possibility.

\section{Relevance to fast-slow matching in the mammal}

Results from the present study support the hypothesis that fast twitch and slow "tonic" chick muscle fibers are selectively innervated by molecularly distinct classes of motoneurons during development. This contrasts with the view, based in large part on regeneration studies in mature mammals (for review, see Vrbova et al., 1995), that fast and slow twitch fibers are nonselectively innervated and that their phenotype is subsequently determined by the electrical activity of the innervating motoneuron. It is clear that in mature mammals, regenerating axons often do not selectively innervate their appropriate fiber type and the fiber-type clumping observed is evidence of the subsequent conversion of fiber types (e.g., Kugelberg et al., 1970; Foehring et al., 1986; Rafuse and Gordon, 1996).

The situation during development, however, may be more similar to what we observed in the chick. First, although fast and slow muscle fibers do not exhibit detectable differences in their physiological properties until quite late (e.g., Buller et al., 1960a; for 
review, see Vrbova et al., 1995), they can be distinguished at early times by their expression of contractile protein isoforms (Condon et al., 1990a; for review, see Donoghue and Sanes, 1994). Additionally, as in the chick, the initial phenotypic differentiation of these early fibers as well as their spatial pattern within the limb does not require neural influence or electrical activity (Condon et al., 1990a,b). Recent evidence also shows that in the adult, some intrinsic differences between fiber types may still exist, setting limits to their ability to be converted by patterns of electrical activity (e.g., Dum et al., 1985; Foehring et al., 1987; Foehring and Munson, 1990; Gordon et al., 1997). Finally, neonatal studies in the rat (Thompson et al., 1984, 1987, 1990), mouse (Fladby and Jansen, 1988, 1990), and rabbit (Gordon and Van Essen, 1985; Soha et al., 1987; Cramer and Van Essen, 1995) have found that motor units are heavily biased toward one fiber type, providing strong evidence to support an initial period of selective recognition (for selective reinnervation during this period, see also Soileau et al., 1987).

As a unifying hypothesis, we suggest that, in both birds and mammals, there is an initial period, based on molecular recognition between motoneurons and muscle fibers, that sets up the basic pattern of fast-slow matching within the limb. This would be followed by a second period during which fine tuning of motor unit properties, including their size, would occur as many more secondary myotubes are generated. During this phase, bidirectional influences between motoneurons and muscle fibers could occur (for review, see Mendell et al., 1994; Vrbova et al., 1995), causing all the fibers within the motor unit to become identical and well matched to the physiological properties of the motoneuron. A test of this hypothesis would be greatly facilitated by the identification of molecular markers to distinguish between fast and slow motoneurons in both birds and mammals at all developmental stages.

\section{REFERENCES}

Appel B, Korzh V, Glasgow E, Thor S, Edlund T, Dawid IB, Eisen JS (1995) Motoneuron fate specification revealed by patterned LIM homeobox gene expression in embryonic zebrafish. Development 121:4117-4125.

Balice-Gordon RJ, Thompson WJ (1988) The organization and development of compartmentalized innervation in rat extensor digitorum longus muscle. J Physiol (Lond) 398:211-231.

Browne KM (1950) The spatial distribution of segmental nerves to striate musculature of the hindlimb of the rat. J Comp Neurol 93:441-455.

Buller AJ, Eccles JC, Eccles RM (1960a) Differentiation of fast and slow muscles in the cat hind limb. J Physiol (Lond) 150:399-416.

Buller AJ, Eccles JC, Eccles RM (1960b) Interactions between motoneurones and muscles in respect of their characteristic speeds of their responses. J Physiol (Lond) 150:417-439.

Burke RE (1981) Motor units: anatomy, physiology, and functional organization. In: Handbook of physiology. The nervous system. Motor control, Sec I, Vol II (Brooks VB, ed), pp 345-422. Bethesda, MD: Williams and Williams.

Butler JB, Cosmos E, Brierley J (1982) Differentiation of muscle fiber types in aneurogenic brachial muscles of the chick embryo. J Exp Zool 224:65-80.

Condon K, Silberstein L, Blau HM, Thompson WJ (1990a) Development of muscle fiber types in the prenatal rat hindlimb. Dev Biol 138:256-274.

Condon K, Silberstein L, Blau H, Thompson WJ (1990b) Differentiation of fiber types in aneural musculature of the prenatal rat hindlimb. Dev Biol 138:275-295.

Cramer KS, Van Essen DC (1995) Maturation of fast and slow motor units during synapse elimination in the rabbit soleus muscle. Dev Biol $171: 16-26$.

Crow MT, Stockdale FE (1986) Myosin expression and specialization among the earliest muscle fibers of the developing avian limb. Dev Biol 113:238-254

Dahm L, Landmesser LT (1988) The regulation of intramuscular nerve branching during normal development and following activity blockade. Dev Biol 130:621-644.

Desai CJ, Gindhart JG, Goldstein LSB, Zinn K (1996) Receptor tyrosine phosphatases are required for motor axon guidance in the Drosophila embryo. Cell 84:599-609.

Desai CJ, Krueger NX, Saito H, Zinn K (1997) Competition and cooperation among receptor tyrosine phosphatases control motoneuron growth cone guidance in Drosophila. Development 124:1941-1952.

Donahue SP, English AW (1987) The role of synapse elimination in the establishment of neuromuscular compartments. Dev Biol 124:481-489.

Donoghue MJ, Sanes JR (1994) All muscles are not created equal. Trends Genet 10:396-401.

Donoghue MJ, Lewis RM, Merlie JP, Sanes JR (1996) The Eph kinase ligand AL-1 is expressed by rostral muscles and inhibits outgrowth from caudal neurons. Mol Cell Neurosci 8:185-198.

Dum RP, O’Donovan MJ, Toop J, Tsairis P, Pinter MJ, Burke RE (1985) Cross-reinnervated motor units in cat muscle. II. Soleus muscle reinnervated by flexor digitorum longus motoneurons. J Neurophysiol 54:837-851.

Ebens A, Brose K, Leonardo ED, Hanson MG, Bladt F, Birchmeier C, Barres BA, Tessier-Lavigne M (1996) Hepatocyte growth factor/ scatter factor is an axonal chemoattractant and a neurotrophic factor for spinal motor neurons. Neuron 17:1157-1172.

English AW, Weeks OI (1984) Compartmentalization of single muscle units in cat lateral gastrocnemius. Exp Brain Res 56:361-368.

Ericson J, Rashbass P, Schedl A, Brenner-Morton S, Kawakami A, van Heyningen V, Jessell TM, Briscoe J (1997) Pax6 controls progenitor cell identity and neuronal fate in response to graded Shh signaling. Cell 90:169-180.

Fambrough D, Goodman CS (1996) The Drosophila beaten path gene encodes a novel secreted protein that regulates defasciculation at motor axon choice points. Cell 87:1049-1058.

Feng TP, Wu WY, Yang FY (1965) Selective reinnervation of a "slow" or "fast" muscle by its original motor supply during regeneration of mixed nerve. Scientia Sin 14:1717-1720.

Fields RD, Itoh K (1996) Neural cell adhesion molecules in activitydependent development and synaptic plasticity. Trends Neurosci 19:473-480.

Fladby T, Jansen JKS (1988) Selective innervation of neonatal fast and slow muscle fibres before net loss of synaptic terminals in the mouse soleus muscle. Acta Physiol Scand 134:561-562.

Fladby T, Jansen JKS (1990) Development of homogeneous fast and slow motor units in the neonatal mouse soleus muscle. Development 109:723-732.

Foehring RC, Munson JB (1990) Motoneuron and muscle-unit properties after long-term direct innervation of soleus muscle by medial gastrocnemius nerve in cat. J Neurophysiol 64:847-861.

Foehring RC, Sypert GW, Munson JB (1986) Properties of selfreinnervated motor units of medial gastrocnemius of cat. I. Long term reinnervation. J Neurophysiol 55:931-946.

Foehring RC, Sypert GW, Munson JB (1987) Motor-unit properties following cross-reinnervation of cat lateral gastrocnemius and soleus muscles with medial gastrocnemius nerve. I. Influence of motoneurons on muscle. J Neurophysiol 57:1210-1226.

Fredette BJ, Landmesser LT (1991a) Relationship of primary and secondary myogenesis to fiber type development in embryonic chick muscle. Dev Biol 143:1-18.

Fredette BJ, Landmesser LT (1991b) A reevaluation of the role of innervation in primary and secondary myogenesis in developing chick muscle. Dev Biol 143:19-35.

Fredette B, Rutishauser U, Landmesser L (1993) Regulation and activity-dependence of $\mathrm{N}$-cadherin, NCAM isoforms, and polyscialic acid on chick myotubes during development. J Cell Biol 123:1867-1888.

Ginsborg BL, Mackay B (1961) A histochemical demonstration of two types of motor innervation in avian skeletal muscle. Bibl Anat 2:174-181.

Goodman CS (1996) Mechanisms and molecules that control growth cone guidance. Annu Rev Neurosci 19:341-377.

Goodman CS, Shatz CJ (1993) Developmental mechanisms that generate precise patterns of neuronal connectivity. Cell [Suppl] 10:77-98.

Gordon H, Van Essen DC (1985) Specific innervation of muscle fiber types in a developmentally polyinnervated muscle. Dev Biol 111:42-50.

Gordon T, Tyreman N, Rafuse VF, Munson JB (1997) Fast-to-slow conversion following chronic low-frequency activation of medial gastrocnemius muscle in cats. I. Muscle and motor unit properties. J Neurophysiol 77:2585-2604. 
Hamburger V (1975) Cell death in the development of the lateral motor column of the chick embryo. J Comp Neurol 160:535-546.

Hamburger V, Hamilton HL (1951) A series of normal stages in the development of the chick embryo. J Morphol 88:49-82.

Hnik P, Jirmanova I, Vyklicky L, Zelena J (1967) Fast and slow muscles of the chick after nerve cross-union. J Physiol (Lond) 193:309-325.

Ho S, O'Donovan MJ (1993) Regionalization and intersegmental coordination of rhythm-generating networks in the spinal cord of the chick embryo. J Neurosci 13:1354-1371.

Hoh JFY (1975) Selective and non-selective reinnervation of fast-twitch and slow-twitch rat skeletal muscle. J Physiol (Lond) 251:791-801.

Jolesz F, Sreter FA (1981) Development, innervation, and activity-pattern induced changes in skeletal muscle. Annu Rev Physiol 43:531-552.

Kernell D (1992) Organized variability in the neuromuscular system: a survey of task-related adaptations. Arch Ital Biol 130:19-66.

Kitsukawa T, Shimizu M, Sanbo M, Hirata T, Taniguchi M, Bekku Y, Yagi T, Fujisawa H (1997) Neuropilin-semaphorin III/D-mediated chemorepulsive signals play a crucial role in peripheral nerve projection in mice. Neuron 19:995-1005.

Krueger NX, Van Vactor D, Wan HI, Gelbart WM, Goodman CS, Saito H (1996) The transmembrane tyrosine phosphatase DLAR controls motor axon guidance in Drosophila. Cell 84:611-622.

Kugelberg E, Edstrom L, Abruzzese M (1970) Mapping of motor units in experimentally reinnervated rat muscle. J Neurol Neurosurg Psychiatry $33: 310-329$.

Lance-Jones C, Landmesser L (1981) Pathway selection by chick lumbosacral motoneurons during normal development. Proc R Soc Lond B Biol Sci 214:1-18.

Landmesser L (1978) The distribution of motoneurones supplying chick hind limb muscles. J Physiol (Lond) 284:371-389.

Landmesser LT (1992) Growth cone guidance in the avian limb: a search for cellular and molecular mechanisms. In: The nerve growth cone (Letourneau PC, Kater SB, Macagno ER, eds), pp 373-385. New York: Raven.

Landmesser LT, O'Donovan MJ (1984a) Activation patterns of embryonic chick hind limb muscles recorded in ovo and in an isolated spinal cord preparation. J Physiol (Lond) 347:189-204.

Landmesser LT, O'Donovan MJ (1984b) The activation patterns of embryonic chick motoneurones projecting to inappropriate muscles. J Physiol (Lond) 347:205-224.

Landmesser L, Dahm L, Schultz K, Rutishauser U (1988) Distinct roles for adhesion molecules during innervation of embryonic chick muscle. Dev Biol 130:645-670.

Landmesser L, Dahm L, Tang J, Rutishauser U (1990) Polyscialic acid as a regulator of intramuscular nerve branching during embryonic development. Neuron 4:655-667.

Laskowski MB, High JA (1989) Expression of nerve-muscle topography during development. J Neurosci 9:175-182.

Laskowski MB, Sanes JR (1987) Topographic mapping of motor pools onto skeletal muscles. J Neurosci 7:252-260.

Matise MP, Lance-Jones C (1996) A critical period for the specification of motor pools in the chick lumbosacral spinal cord. Development 122:659-669.

McLennan IS (1983) The development of the pattern of innervation in chicken hindlimb muscles: evidence for specification of nerve-muscle connections. Dev Biol 97:229-238.

Mendell LM, Collins WF, Munson JB (1994) Retrograde determination of motoneuron properties and their synaptic input. J Neurobiol 25:707-721.

Mitchell KJ, Doyle JL, Serafini T, Goodman CS, Dickson BJ (1996) Genetic analysis of netrin genes in Drosophila: netrins guide CNS commissural axons and peripheral motor axons. Neuron 17:203-215.

O'Donovan MJ (1989) Motor activity in the isolated spinal cord of the chick embryo: synaptic drive and firing pattern of single motoneurons. J Neurosci 9:943-958.

Oppenheim RW (1991) Cell death during development of the nervous system. Annu Rev Neurosci 14:453-501.

Phillips WD, Bennett MR (1984) Differentiation of fiber types in wing muscles during development: effect of neural tube removal. Dev Biol 106:457-468.

Rafuse VF, Gordon T (1996) Self-reinnervated cat medial gastrocnemius muscles. II. Analysis of the mechanisms and significance of fiber type grouping in reinnervated muscles. J Neurophysiol 75:282-297.

Rafuse VF, Landmesser L (1996) Contractile activity regulates isoform expression and polyscialylation of NCAM in cultured myotubes: involvement of $\mathrm{Ca}^{2+}$ and protein kinase C. J Cell Biol 132:969-983.

Rafuse VF, Milner LD, Landmesser LT (1996) Selective innervation of fast and slow muscle regions during early chick neuromuscular development. J Neurosci 16:6864-6877.

Romer AS (1927) The development of the thigh musculature of the chick. J Morphol 43:347-385.

Schroeter S, Tosney KW (1991) Spatial and temporal patterns of muscle cleavage in the chick thigh and their value as criteria for homology. Am J Anat 191:325-350.

Sernagor E, Chub N, Ritter A, O'Donovan MJ (1995) Pharmacological characterization of the rhythmic synaptic drive onto lumbosacral motoneurons in the chick embryo spinal cord. J Neurosci 15:7452-7464.

Sholomenko GN, O'Donovan MJ (1995) Development and characterization of pathways descending to the spinal cord in the embryonic chick. J Neurophysiol 73:1223-1233.

Soha JM, Yo C, Van Essen DC (1987) Synapse elimination by fiber type and maturational state in rabbit soleus muscle. Dev Biol 123:136-144.

Soileau LC, Silberstein L, Blau HM, Thompson WJ (1987) Reinnervation of muscle fiber types in the newborn rat soleus. J Neurosci 7:4176-4194.

Swett JE, Eldred E, Buchwald JS (1970) Somatotopic cord-to-muscle relations in efferent innervation of cat gastrocnemius. Am J Physiol 219:762-766.

Tang J, Landmesser L, Rutishauser U (1992) Polyscialic acid influences specific pathfinding by avian motoneurons. Neuron 8:1031-1044.

Tang J, Rutishauser U, Landmesser L (1994) Polyscialic acid regulates growth cone behavior during sorting of motor axons in the plexus region. Neuron 13:405-414.

Thompson WJ, Sutton LA, Riley DA (1984) Fiber type composition of single motor units during synapse elimination in neonatal rat soleus muscle. Nature 309:709-711.

Thompson WJ, Soileau LC, Balice-Gordon RJ, Sutton LA (1987) Selective innervation of types of fibers in developing rat muscle. J Exp Biol 132:249-263.

Thompson WJ, Condon K, Astrow SH (1990) The origin and selective innervation of early muscle fiber types in the rat. J Neurobiol 21:212-222.

Tosney KW, Landmesser LT (1985) Specificity of early motoneuron growth cone outgrowth in the chick embryo. J Neurosci 5:2336-2344.

Vogel MW (1987) Activation patterns of embryonic chick lumbosacral motoneurones following large spinal cord reversals. J Physiol (Lond) 389:491-512.

Vogel MW, Landmesser L (1987) Distribution of fiber types in embryonic chick limb muscles innervated by foreign motoneurons. Dev Biol 119:481-495.

Vrbova G, Gordon T, Jones R (1995) Nerve-muscle interaction. London: Chapman and Hall.

Wang HU, Anderson DJ (1997) Eph family transmembrane ligands can mediate repulsive guidance of trunk neural crest migration and motor axon outgrowth. Neuron 18:383-396.

Weeks OI, English AW (1985) Compartmentalization of the cat lateral gastrocnemius motor nucleus. J Comp Neurol 235:255-267. 